\title{
On the potential and challenges of laser-induced thermal acoustics for experimental investigation of macroscopic fluid phenomena
}

\author{
Christoph Steinhausen $^{1}$ (D) $\cdot$ Valerie Gerber $^{1} \cdot$ Andreas Preusche $^{2} \cdot$ Bernhard Weigand $^{1} \cdot$ Andreas Dreizler $^{2} \cdot$ \\ Grazia Lamanna ${ }^{1}$
}

Received: 5 June 2020 / Revised: 19 October 2020 / Accepted: 22 October 2020

(c) The Author(s) 2020

\begin{abstract}
Mixing and evaporation processes play an important role in fluid injection and disintegration. Laser-induced thermal acoustics (LITA) also known as laser-induced grating spectroscopy (LIGS) is a promising four-wave mixing technique capable to acquire speed of sound and transport properties of fluids. Since the signal intensity scales with pressure, LITA is effective in high-pressure environments. By analysing the frequency of LITA signals using a direct Fourier analysis, speed of sound data can be directly determined using only geometrical parameters of the optical arrangement no equation of state or additional modelling is needed at this point. Furthermore, transport properties, like acoustic damping rate and thermal diffusivity, are acquired using an analytical expression for LITA signals with finite beam sizes. By combining both evaluations in one LITA signal, we can estimate mixing parameters, such as the mixture temperature and composition, using suitable models for speed of sound and the acquired transport properties. Finally, direct measurements of the acoustic damping rate can provide important insights on the physics of supercritical fluid behaviour.
\end{abstract}

\section{Graphic Abstract}

\section{On the potential and challenges of laser induced thermal acoustics for experimental investigation of macroscopic fluid phenomena}

Laser induced thermal acoustic

(LITA) with focused beams
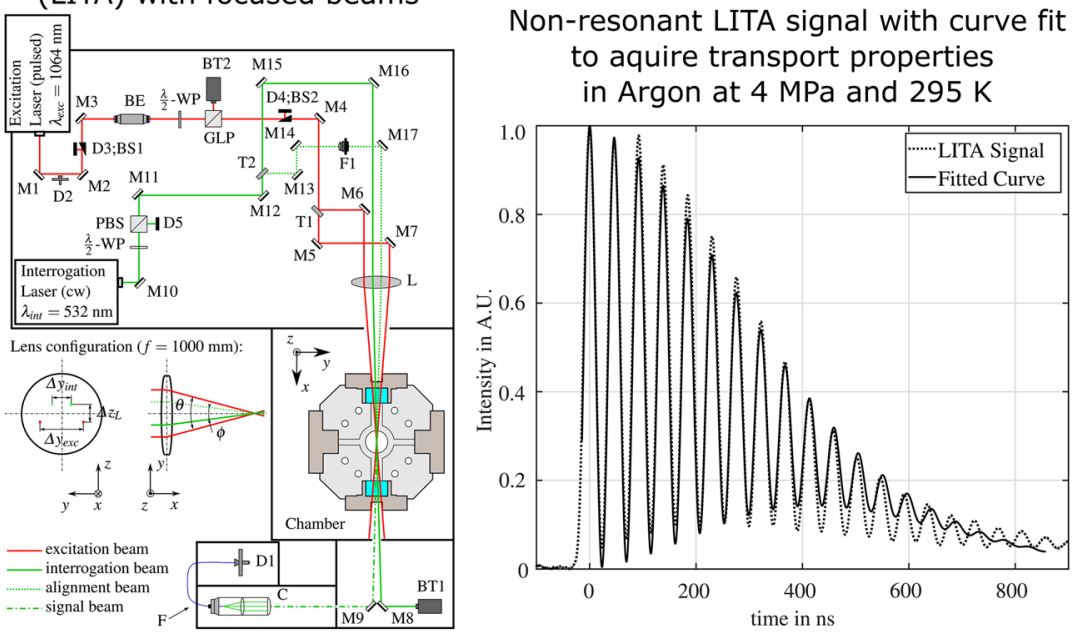

Acoustic damping rate ratio for Argon over pressure at room temperature

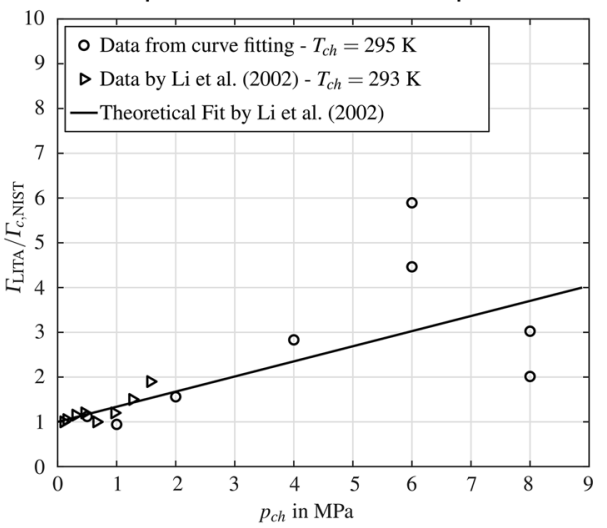

Extended author information available on the last page of the article

List of symbols

Latin characters

$A_{P 1, P 2}$ Complex amplitudes of the acoustic waves (-)

$A_{T} \quad$ Complex amplitudes of the thermal grating (-) 
$B \quad$ Amplitude of harmonic oscillation (-)

$C \quad$ Amplitude of harmonic oscillation (-)

$D_{T} \quad$ Thermal diffusivity $\quad\left(\mathrm{m}^{2} \mathrm{~s}^{-1}\right)$

$E_{\text {exc }} \quad$ Pulse energy of the excitation

beams $\quad\left(\mathrm{kg} \mathrm{m}^{2} \mathrm{~s}^{-2}\right)$

I Signal intensity (-)

$P_{1,2} \quad$ Complex parameter to compute $\Psi(t)$;

$P_{1,2}=A_{P 1, P 2} \Sigma_{P 1, P 2} \quad(-)$

$P_{\text {int }} \quad$ Power of the interrogation beam $\quad\left(\mathrm{kg} \mathrm{m}^{2} \mathrm{~s}^{-3}\right)$

$T \quad$ Complex parameter to compute $\Psi(t)$;

$T=A_{T} \Sigma_{T} \quad(-)$

$T_{c h} \quad$ Fluid temperature in measurement chamber $(\mathrm{K})$

$T_{\text {mix }} \quad$ Local mixing temperature $(\mathrm{K})$

$U_{\Theta} \quad$ Dimensionless modulation depth of thermalization grating (-)

$U_{e P} \quad$ Dimensionless modulation depth of electrostriction grating (-)

$c_{p} \quad$ Specific isobaric heat capacity $\quad\left(\mathrm{m}^{2} \mathrm{~s}^{-2} \mathrm{~K}^{-1}\right)$

$c_{s} \quad$ Speed of sound $\left(\mathrm{m} \mathrm{s}^{-1}\right)$

$f \quad$ Focal length (m)

$j \quad$ Indicator related to fluid behaviour; $j=1$ : resonant; $j=2$ : non-resonant $\quad(-)$

$\begin{array}{ll}p & \text { Pressure } \quad\left(\mathrm{kg} \mathrm{m}^{-1} \mathrm{~s}^{-2}\right) \\ q & \text { Magnitude of the grating vector; }\end{array}$

$q=2 \pi / \Lambda \quad\left(\mathrm{m}^{-1}\right)$

$s \quad$ Specific entropy $\left(\mathrm{m}^{2} \mathrm{~s}^{-2} \mathrm{~K}^{-1}\right)$

Time (s)

$t_{0} \quad$ Time of laser pulse (s)

$v \quad$ Fluid velocity component in $y$-direction $\quad\left(\mathrm{m} \mathrm{s}^{-1}\right)$

$w \quad$ Fluid velocity component in $z$-direction $\quad\left(\mathrm{m} \mathrm{s}^{-1}\right)$

$x_{F l} \quad$ Local mole fraction of a fluid in a mixture (-)

$x \quad$ Cartesian coordinate (m)

$y \quad$ Cartesian coordinate (m)

$z \quad$ Cartesian coordinate (m)

\section{Greek characters}

$\Delta y \quad$ Beam distance in front of lens (m)

$\Delta z_{L} \quad$ Distance between the interrogation and excitation beams in front of lens $(\mathrm{m})$

$\Gamma \quad$ Acoustic damping rate $\quad\left(\mathrm{m}^{2} \mathrm{~s}^{-1}\right)$

$\Gamma_{c} \quad$ Classical acoustic damping rate; bulk viscosities are neglected $\quad\left(\mathrm{m}^{2} \mathrm{~s}^{-1}\right)$

$\Gamma_{m} \quad$ Pressure corrected classical acoustic damping rate according to the theoretical and empirical considerations of Li et al. (2002) $\quad\left(\mathrm{m}^{2} \mathrm{~s}^{-1}\right)$

$\Lambda \quad$ Grid spacing of the optical interference pattern (m)

$\Phi \quad$ Crossing angle of interrogation beam (rad)

$\Psi(t) \quad$ Time-dependent dimensionless diffraction efficiency of a LITA signal (-)
$\Sigma_{P 1, P 2}$ Complex parameter related to the damping of oscillations (-)

$\Sigma_{T} \quad$ Complex parameter related to the damping of the signal (-)

$\Theta \quad$ Crossing angle of excitation beam (rad)

$\alpha \quad$ Calibration constant $\quad\left(\mathrm{s}^{2} \mathrm{~kg}^{-1} \mathrm{~m}^{-2}\right)$

$\beta \quad$ Decay rate $\left(\mathrm{s}^{-1}\right)$

$\bar{\eta} \quad$ Misalignment length scales in $y$-direction

(m)

$\gamma \quad$ Specific heat ratio (-)

$\gamma_{n \Theta} \quad$ Rate of excited-state energy decay not caused by thermalization $\left(\mathrm{s}^{-1}\right)$

$\gamma_{\Theta} \quad$ Rate of excited-state energy decay caused by thermalization $\left(\mathrm{s}^{-1}\right)$

$\kappa \quad$ Thermal conductivity $\left(\mathrm{kg} \mathrm{m} \mathrm{s}^{-3} \mathrm{~K}^{-1}\right)$

$\lambda \quad$ Wavelength (m)

$\mu_{v} \quad$ Dynamic bulk viscosity $\quad\left(\mathrm{kg} \mathrm{m}^{-1} \mathrm{~s}^{-1}\right)$

$\mu_{s} \quad$ Dynamic shear viscosity $\quad\left(\mathrm{kg} \mathrm{m}^{-1} \mathrm{~s}^{-1}\right)$

$v \quad$ Dominating frequency of the LITA

signal $\left(\mathrm{s}^{-1}\right)$

$\omega \quad$ Gaussian half-width of excitation beams (m)

$\varrho \quad$ Mass density $\left(\mathrm{kg} \mathrm{m}^{-3}\right)$

$\sigma \quad$ Gaussian half-width of interrogation beam

(m)

$\tau \quad$ Laser pulse length (s)

$v \quad$ Angular frequency $\quad\left(\mathrm{s}^{-1}\right)$

$v_{0} \quad$ Natural angular frequency associated with speed of sound $\left(\mathrm{s}^{-1}\right)$

$\bar{\zeta} \quad$ Misalignment length scales in $z$-direction

\section{Subscripts}

Ar Related to fluid: argon

$B P \quad$ Related to beam profiler measurement

DFT Related to calculations using a direct Fourier transformation

LITA Related to measurement using LITA

N2 Related to fluid: nitrogen

NIST Related to theoretical calculations using NIST database by Lemmon et al. (2018)

atm Unit of pressure used: atmospheres

c Related to properties at the critical point

cal Related to calibration

ch Related to condition in measurement chamber

exc Related to excitation beam

int Related to interrogation beam

mix Related to local condition of mixture

$r \quad$ Reduced properties scaled with the properties related to the critical point

th Related to theoretical calculations using data sheet specifications

\section{Miscellaneous characters}

$\mathcal{O}(\cdot) \quad$ Order of magnitude

$(\cdot)^{*} \quad$ Complex conjugate 


$\begin{array}{ll}\text { Abbreviations } \\ \text { DFT } & \text { Direct Fourier transformation } \\ \text { GLP } & \text { Glan-Laser polarizer } \\ \text { LAR } & \text { Least-Absolute Residuals } \\ \text { LIGS } & \text { Laser-induced (transient) grating spectroscopy } \\ \text { LITA } & \text { Laser-induced thermal acoustics } \\ \text { MM } & \text { Multi-mode fibre with diameter of } 25 \mu \mathrm{m} \\ \text { NIST } & \text { National Institute of Standards and Technology } \\ \text { PBS } & \text { Polarizing beam splitter } \\ \text { SBS } & \text { Stimulated Brillouin scattering } \\ \text { SM } & \text { Single-mode fibre with diameter of } 4 \mu \mathrm{m} \\ \text { STBS } & \text { Stimulated thermal Brillouin scattering } \\ \text { STRS } & \text { Stimulated (thermal) Rayleigh scattering } \\ \text { WP } & \lambda / 2 \text {-wave plate }\end{array}$

\section{Introduction}

Fluid injection, disintegration, and subsequent evaporation are of high importance for a stable and efficient combustion. Especially for high pressures exceeding the critical value of the injected fluids, mixing and evaporation processes as well as fundamental changes in fluid behaviour are not yet fully understood. The latter have received increased attention in the past decade, as the recently published literature shows (Falgout et al. 2016; Müller et al. 2016; Baab et al. 2016, 2018; Crua et al. 2017). Since the main objectives are evaporation and disintegration processes of liquid fluids at pressures and temperatures either close to or exceeding their critical points, quantitative data for validation of numerical simulations have recently become a research concern with increasing interest (Bork et al. 2017; Lamanna et al. 2018; Steinhausen et al. 2019; Stierle et al. 2020; Nomura et al. 2020; Lamanna et al. 2020; Qiao et al. 2020). Microscopic investigations by Santoro and Gorelli (2008), Simeoni et al. (2010) as well as Bencivenga et al. (2009) made it possible to distinguish various regions above the critical pressure, as is depicted in Fig. 1. At supercritical pressures, the region between the critical isotherm and the Widom line, which is characterized by the maximum in specific isobaric heat capacity, is identified as liquid-like. Indeed, it preserves large densities and sound dispersion (Simeoni et al. 2010; Bencivenga et al. 2009), while exhibiting the molecular structure of a gas (Santoro and Gorelli 2008). In contrast, regions with supercritical temperatures right of the Widom line are gas-like, as propagation of sound waves at the adiabatic speed of sound is recovered (Simeoni et al. 2010). In this context, the area between the critical isotherm and the Widom line can be denoted as the supercritical region, because it exhibits dynamical and physical properties intermediate between gas and liquid states. The relevance of these microscopic findings on the dynamic behaviour of

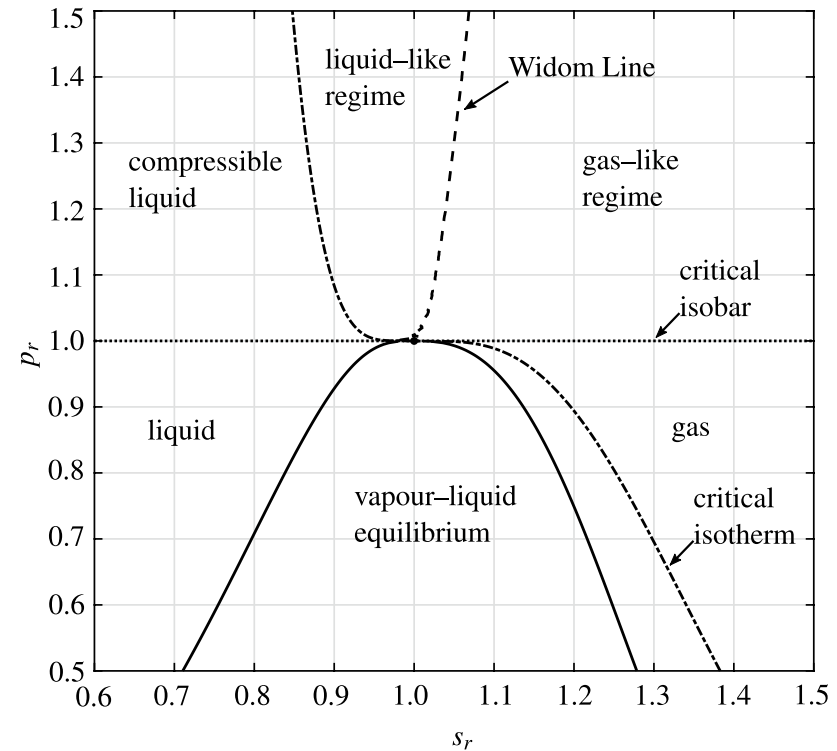

Fig. 1 Thermodynamic fluid states based on microscopic investigations; $p_{r}=p / p_{c}$ : reduced pressure; $s_{r}=s / s_{c}$ : reduced entropy; reduced properties are scaled with the properties related to the critical point; Widom line: line of maxima in specific isobaric heat capacity; thermodynamic data are taken from Lemmon et al. (2018)

supercritical fluids at macroscopic scale remains till today poorly understood. In Sect. 2, it is shown how the acoustic damping rate enables to disclose the interrelated nature of sound dispersion at microscopic and macroscopic scales. At this stage, it is important to point out that the current macroscopic description of supercritical states is mainly focused on the selection of accurate equation of states. The latter are capable to describe the continuous fluid transformation in terms of density changes and the singularities in terms of some physical properties (heat capacity, isothermal compressibility, etcetera) across the Widom line. This approach, however, may not be sufficient for a correct description of the dynamical behaviour of supercritical fluids, as currently suggested by the microscopic investigations.

The previous consideration provides the motivation for the present work, where emphasis is placed on the measurement of speed of sound $\left(c_{s}\right)$, thermal $\left(D_{T}\right)$, and viscous $(\Gamma)$ relaxation constants, identified as key parameters to enable macroscopic investigations of these different fluid regions. In addition, these macroscopic fluid properties, such as speed of sound, acoustic damping, and thermal diffusivity, enable a quantitative comparison of injection studies with analytical and numerical data, as has been shown for speed of sound measurements in high-pressure jets by Baab et al. (2018). The speed of sound data of the mixture was acquired using homodyne laser-induced thermal acoustics (LITA). A detailed description of the used experimental setup can be found in the work of Förster (2016). A comprehensive 
review on gas-phase diagnostics using laser-induced transient grating spectroscopy (LIGS) is provided by Stampanoni-Panariello et al. (2005b).

LIGS, LITA or similar techniques are mostly used to determine transport properties in quiescent environments, where high spatial resolution is not the focus in the investigation. However, LITA is indeed sensitive to small-scale processes, as investigation of the speed of sound data in multi-component jet mixing at high pressures by Baab et al. (2018) has shown. Kimura et al. (1995) measured transport properties of high-pressure fluids, namely carbon dioxide and trifluoromethane, using LIGS. The study focused mainly on determination of thermal diffusion, mass diffusion, and sound propagation in the vicinity of the critical point. In the same research group, thermal diffusion and sound propagation of binary mixtures of carbon dioxide and a hexafluorophosphate were investigated by Demizu et al. (2008). Latzel and Dreier (2000) investigated heat conduction, speed of sound data, as well as viral coefficients of gaseous mixtures at pressures up to $50 \mathrm{MPa}$ by analysing the acoustic oscillations and the long-term decay of a near-infrared LIGS signal. Vibrational energy relaxation of azulene was studied in super-critical states by Kimura et al. (2005a) and for liquid solvents by Kimura et al. (2005b). An investigation of acoustic damping rates in pure gases was presented by $\mathrm{Li}$ et al. (2002) analysing the temporal behaviour of transient grating spectroscopy. The investigations included different gases at pressures up to $25 \mathrm{~atm}$ at room temperature. Li et al. (2002) compared these findings with classical acoustic theory and derived a linear pressure dependency for the measured acoustic damping rate. Li et al. (2005) later proposed a binary mixture model to determine the acoustic damping rate for binary atomic species. Note that all the previously reviewed studies measure transport properties using an optical arrangement with unfocused beams for grid excitation. This leads to a measurement volume with an order of magnitude $\mathcal{O}\left(10^{1} \mathrm{~mm}\right)$ in diameter and $\mathcal{O}\left(10^{2} \mathrm{~mm}\right)$ in length and hence a poor spatial resolution.

To utilize LITA as a reliable tool for experimental investigation in jet disintegration or droplet evaporation studies, a high spatial resolution is imperative. Studies by Baab et al. (2016), Baab et al. (2018), and Förster et al. (2018) already showed the capability of acquiring quantitative speed of sound data in jet disintegration. Especially to be emphasised are the investigations by Baab et al. (2018), which demonstrated the potential of acquiring speed of sound data for multi-component jet mixing at high pressures in the near nozzle region. The purpose of this study is to present the calibration and validation processes needed for the extraction of speed of sound data, acoustic damping rates, as well as thermal diffusivities using LITA with a spatial resolution with an order of magnitude $\mathcal{O}\left(10^{-1} \mathrm{~mm}\right)$ in diameter and $\mathcal{O}\left(10^{0} \mathrm{~mm}\right)$ in length in a high-pressure and high-temperature environment for resonant and non-resonant fluids.

\section{Theoretical consideration on the relevance of laser-induced thermal acoustic in supercritical mixture studies}

The LITA (or LIGS) technique provides an excellent opportunity to measure independently and simultaneously speed of sound data and acoustic damping rates. The implications of these measurements are twofold. First, measuring acoustic damping rates allows to assess whether sound dispersion in supercritical fluids is significant and provides the possibility to indirectly measure bulk viscosities, which are mainly responsible for sound dispersion. Additionally, bulk viscosities will enable the improvement of models for the stress tensor and the kinetic energy dissipation in supercritical fluid flow simulations. Second, if both speed of sound and acoustic damping rate are measured, a set of independent equations can be derived to extract local mixing parameters, like temperature and composition of a binary mixture.

The possibility to estimate mixing parameters using laser induced thermal acoustics was shown by Li et al. (2005) for binary mixtures of monoatomic species. Using transient grating spectroscopic $\mathrm{Li}$ et al. (2005) were able to derive the mole fraction of a Helium-Argon mixture. As we will later show in the post-processing section of this work (Sect. 3.2.2), analysing the temporal evolution of a detected LITA signal enables the determination of three transport properties, namely the speed of sound $c_{s}$, the acoustic damping rate $\Gamma$, and, in case of a resonant fluid behaviour, the thermal diffusivity $D_{T}$. Whereas thermal diffusivity and speed of sound are well-known transport properties and accessible using the NIST database by Lemmon et al. (2018), acoustic damping rates have to be modelled in more detail. Dissipation of a sound waves energy is mainly caused by internal friction and heat conduction. The damping rate of an acoustic wave is, therefore, dependent on the viscosity and thermal conductivity (Li et al. 2002). Using the theoretical description by Hubschmid et al. (1995), the acoustic damping rate $\Gamma$ depends on both shear viscosity $\mu_{s}$ and bulk viscosity $\mu_{v}$, and can be modelled as:

$\Gamma=\frac{1}{2 \varrho}\left[\frac{4}{3} \mu_{s}+\mu_{v}+(\gamma-1) \frac{\kappa}{c_{p}}\right]$,

where $\rho$ is the fluid density, $\gamma$ the specific heat ratio, $\kappa$ the thermal conductivity, and $c_{p}$ the specific isobaric heat capacity. At atmospheric conditions, bulk viscosities are neglectable compared to shear viscosities; using this assumptions, we can calculate the classical acoustic damping rate $\Gamma_{c}$ as used by Li et al. (2002): 
$\Gamma_{c}=\frac{1}{2 \varrho}\left[\frac{4}{3} \mu_{s}+(\gamma-1) \frac{\kappa}{c_{p}}\right]$.

$\Gamma_{c}$ predicts accurate damping rates for monoatomic substances at low pressures. For pressures up to $2.5 \mathrm{MPa}$ at room temperature, Li et al. (2002) estimated the pressure dependence of measured acoustic damping rate with respect to the classical solution. A linear dependence with a fluid-dependent slope was found. For nitrogen and argon, the measured acoustic damping rate is expressed in (3). Note that the unit of pressure used by Li et al. (2002) is atmospheres:

$\Gamma_{m, A r}=\Gamma_{c}\left(1 / 30 p_{a t m}+1\right)$

$\Gamma_{m, N 2}=\Gamma_{c}\left(1 / 6 p_{a t m}+1\right)$.

As mentioned in the Introduction, for mixing processes, e.g., high-pressure turbulent jets, transport properties can be used to derive the local mixing state of macroscopic fluid phenomena. These mixing states are defined by the local temperature of the mixture $T_{\text {mix }}$, the local mole fraction of the fluid $x_{F l, m i x}$, and the overall pressure in the measurement chamber $p_{c h}$. The local transport properties speed of sound $c_{s, m i x}$, acoustic damping rate $\Gamma_{m i x}$, and thermal diffusivity $D_{T, \text { mix }}$ of the mixture can be expressed in the following way:

$$
\begin{aligned}
c_{s, \text { mix }}\left(p_{c h}, T_{\text {mix }}, x_{F l, m i x}\right) & =c_{s, \text { LITA }} \\
\Gamma_{\text {mix }}\left(p_{c h}, T_{\text {mix }}, x_{F l, m i x}\right) & =\Gamma_{\text {LITA }} \\
D_{T, \text { mix }}\left(p_{c h}, T_{\text {mix }}, x_{F l, m i x}\right) & =D_{T, \text { LITA }} .
\end{aligned}
$$

Using a controlled environment where the pressure in the measurement chamber $p_{c h}$ is known, the local mixing temperatures $T_{m i x}$ and local mole fraction of the fluid $x_{F l, m i x}$ are the only unknown fluid properties of the investigated mixture. After careful validation in well-known binary gas-fluid mixtures, this should enable us to determine the desired mixing parameters when coupling them to non-ideal mixture data, e.g., Lemmon et al. (2018). By measuring LITA signals using an optical arrangement with focused beams, it would, therefore, be possible to determine the local mixing temperature and mole fraction simultaneously. This would enable us to study mixing and evaporation processes in highpressure jet mixing or droplet evaporation in the vicinity of the critical point.

To evaluate whether the phenomenon of sound dispersion in supercritical fluids is significant, we first have to understand the behaviour of the relaxation of an electrostriction grating. The latter can be assimilated to a damped harmonic oscillator, which admits a general solution of the following type:

$I(t)=B \exp \{-\beta t-i v t\}+C \exp \{-\beta t+i v t\}$,

where $I$ denotes the signal intensity, $t$ denotes the time, $B$ and $C$ are dimensionless amplitudes, $\beta^{-1}$ is the characteristic decay time of the oscillation's amplitude, and $v$ is the angular frequency. Note that the decay rate $\beta$ is directly proportional to the damping constant, like the acoustic damping rate for acoustic waves. With reference to laser-induced gratings, it was found by Stampanoni-Panariello et al. (2005a) that $\beta=q^{2} \Gamma$. Here, $q$ denotes the magnitude of the grating vector. The frequency of the counter-propagating acoustic waves can be, therefore, expressed as (see Hubschmid et al. (1996)):

$v=\sqrt{v_{0}^{2}-q^{4} \Gamma^{2}}$,

where $v_{0}$ is the natural frequency, which is associated with the adiabatic speed of sound $c_{s}$ in the following way:

$v_{0}=q c_{s}$.

For a system with a small damping constant $\left(v_{0} \gg \beta\right)$, it follows that the frequency of oscillation is close to the undamped natural frequency $v=v_{0}=$ const.. The implications of Eq. 6 are twofold. First, it shows that the local sound speed depends indeed upon the acoustic damping rate. Only if the latter is negligible, we recover the well-known condition that sound waves propagate at the adiabatic speed of sound for low-pressure gases. Second, it follows that the local speed of sound is a function of the excitation grating vector. This effect is known as sound dispersion and is commonly observed in liquids, as has been shown by Mysik (2015). For supercritical fluids, Simeoni et al. (2010) demonstrated that a significant sound dispersion could be observed in the region comprised between the critical isotherm and the Widom line. However, the probing length scale was much smaller (X-ray scattering), thus resulting in larger $q$ values and, therefore, larger frequency dispersions.

Following the procedure adopted by Mysik (2015) for liquids, the bulk viscosity can be measured as deviation between the measured acoustic damping $\Gamma$ in Eq. 1 and the classical model $\Gamma_{c}$ in Eq. 2. In liquids, high values of the bulk viscosity are mainly responsible for the observed sound dispersion. LITA measurements, therefore, will enable to verify whether this behaviour is valid also for supercritical fluids.

\section{Experimental facility and measurement technique}

Investigations for this study are performed in well-controlled quiescent conditions. Three different atmospheres, namely nitrogen with a purity of $99.999 \%$, argon with a purity of $99.998 \%$, and carbon dioxide with a purity of $99.995 \%$, are studied. The optical setup is adapted from the one described in Baab et al. (2016) and Förster et al. (2015). 


\subsection{Pressure chamber}

Experimental investigations are performed using a heatable high-pressure, high-temperature chamber. The latter is designed for phenomenological as well as statistical investigations of free-falling droplets in a near-critical environment. For the presented study, the droplet generator on top of the chamber is replaced with a closed lid. The operating condition for nitrogen range between $p_{c h}=2$ and $8 \mathrm{MPa}$ for temperatures up to $T_{c h}=700 \mathrm{~K}$. For argon and carbon dioxide, the operating pressures vary between 0.5 and $8 \mathrm{MPa}$ for temperatures up to $600 \mathrm{~K}$. Before each set of experiments, the chamber is carefully evacuated to ensure no contamination from previous investigations. The experimental setup is operated as a continuous-flow reactor. The mass flow into the chamber is hereby controlled using a heat-capacity-based mass flow controller (Bronkhorst) for nitrogen and argon as well as a Coriolis-based mass flow controller for carbon dioxide. Note that carbon dioxide is pressurized beforehand using a pneumatic-driven piston compressor. Pressurized fluids are supplied from two sides on top of the chamber through an annular orifice. The pressure inside the chamber is controlled using a pneumatic valve at the system exhaust (Badger Meter). Since the derivation of the present equations relies on the assumption of negligible flow velocities, it is important to emphasise that the used mass flow does not exceed $2.5 \mathrm{~kg} / \mathrm{h}$ for carbon dioxide and $1.25 \mathrm{~kg} / \mathrm{h}$ for argon and nitrogen, which leads to flow velocities below $0.04 \mathrm{~m} / \mathrm{s}$.

The chamber is constructed of heat-resistant stainless steel (EN-1.4913). Eight UV-transparent quartz windows at two different heights are placed at an angle of $90^{\circ}$ to each other ensuring optical accessibility. Eight heating cartridges are vertically inserted in the chamber body. Additionally, a heating plate with four cartridges is placed below the chamber. All heaters are controlled using type-K thermocouples in the chamber body as well as the heater cartridges. The chamber encloses a cylindrical core with a diameter of $40 \mathrm{~mm}$ and a height of $240 \mathrm{~mm}$. For thermal insulation, a mineral-based silicate (SILCA $250 \mathrm{KM}$ ) is used. The bottom of the heating plate is insulated using a vermiculite plate. Vertical and horizontal sectional drawings of the chamber are depicted in Fig. 2. For pressure measurement inside the chamber, a temperature-compensated pressure transducer (Keller $35 \mathrm{X}$ HTC) with an uncertainty rated at $\pm 0.1 \mathrm{MPa}$ is chosen. The pressure transducer is located at the chamber exhaust. Temperature measurements inside the chamber take place at three different heights with miniaturized resistance thermometers penetrating the metal core. Since the uncertainty of these resistance thermometers is temperaturedependent, the measurement uncertainties are calculated for each condition separately. Both temperature and pressure are logged continuously.

\subsection{Laser-induced thermal acoustics}

LITA, also more generally referred to as LIGS, is discussed in detail in literature. A theoretical approach describing the generation of the laser-induced grating as well as the inherent phonon-photon and thermon-photon interaction can be found in Cummings et al. (1995) and Stampanoni-Panariello et al. (2005a). Note that the analytical expression presented by Stampanoni-Panariello et al. (2005a) is only valid for infinite beam sizes, whereas Cummings et al. (1995) takes finite beam sizes into account. In the limit of infinite beam sizes, both theories merge. Schlamp et al. (1999) extended
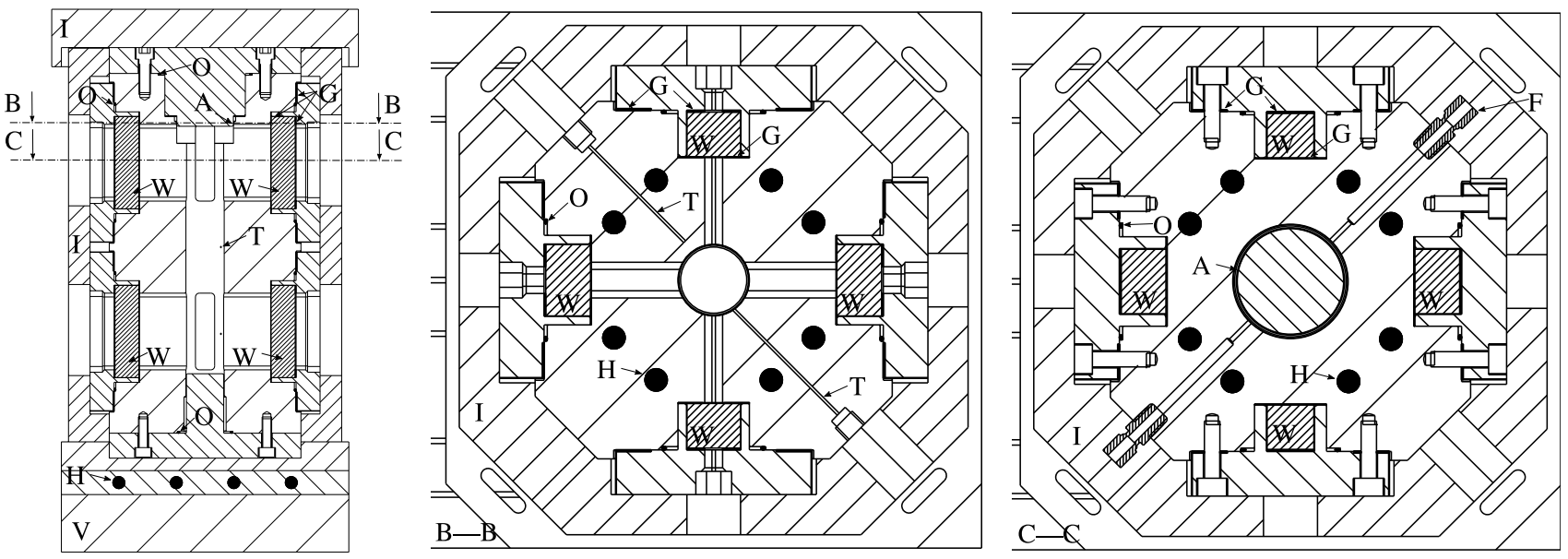

Fig. 2 Horizontal and vertical sections of the high-pressure chamber. Left: vertical section through pressure chamber. Centre (section B-B): horizontal section through pressure chamber at centre of first window. Right (section $\mathrm{C}-\mathrm{C}$ ): horizontal section through pressure chamber at fluid inlet and annular orifice. A: annular orifice; F: fluid inlet; G: graphite gaskets; H: heating cartridges; I: thermal insulation; $\mathrm{O}$ : Willis O-rings; $\mathrm{T}$ : resistance thermometer; V: vermiculite plate; $\mathrm{W}$ : quartz windows 
the theory presented by Cummings et al. (1995) to account for beam misalignment and flow velocities.

LITA occurs due to the non-linear interaction of matter with an optical interference pattern. The latter is introduced by two short-pulsed excitation laser beams, which are crossed using the same direction of linear polarization to produce a spatially periodic modulated polarization/light intensity distribution. The resulting changes in the optical properties of the investigated fluids are interrogated using a third input wave. The third wave originates from a second laser source and is scattered by the spatially periodic perturbations within the measurement volume. Depending on the absorption cross-section of the investigated fluid, changes in optical properties result from different processes. For non-resonant substances, pure electrostriction is observed, whereas in resonant substances, simultaneously, an additional thermal grating is produced. Eichler et al. (1986) distinguishes three dominant forms of light scattering important for LITA. Light scattering from a non-resonant grating can be referred to as stimulated Brillouin scattering (SBS), whereas scattering from a resonant thermal grating depends on the thermalization time. In case of fast energy exchange, stimulated thermal Brillouin scattering is observed (STBS). For slow energy exchange, stationary density modulations emerge, which are referred to as stimulated (thermal) Rayleigh scattering (STRS).

\subsubsection{Optical setup}

The optical arrangement used for the presented investigations is depicted in Fig. 3. For excitation, a pulsed Nd:YAG laser (Spectra Physics QuantaRay: $\lambda_{\text {exc }}=1064 \mathrm{~nm}$, $\tau_{\text {pulse }}=10 \mathrm{~ns}, 30 \mathrm{GHz}$ line width) is used. To ensure stable and reproducible conditions, the excitation laser is set to a pulse energy of $150 \mathrm{~mJ}$, which is continuously measured by a pyroelectric sensor (D3). The energy of the excitation pulse is subsequently controlled using a $\lambda / 2$-wave plate (WP) together with a Glan-Laser polarizer (GLP) and continuously observed by a pyroelectric sensor (D4; Thorlabs). The pulse energy used for investigation is adjusted to values between 18 and $50 \mathrm{~mJ}$. The GLP additionally ensures polarization of the excitation beam, which is split by a beam splitter (T1) into two excitation beams.

The interrogation laser source is provided using a continuous-wave DPSS laser (Coherent Verdi V8, $\lambda_{i n t}=532 \mathrm{~nm}$, $5 \mathrm{MHz}$ line width). The power of the interrogation laser is adjusted to ensure a good signal-to-noise ratio and varies from 0.1 to $8.5 \mathrm{~W}$. Note that, to ensure stable power output at low power settings, the beam power is reduced using a polarizing beam splitter (PBS) together with a $\lambda / 2$-WP.

A forward folded BOXCARS configuration is used to arrange all beams and achieve phase matching. An ARcoated lens ( $f=1000 \mathrm{~mm}$ at $532 \mathrm{~nm})$ is utilized to focus

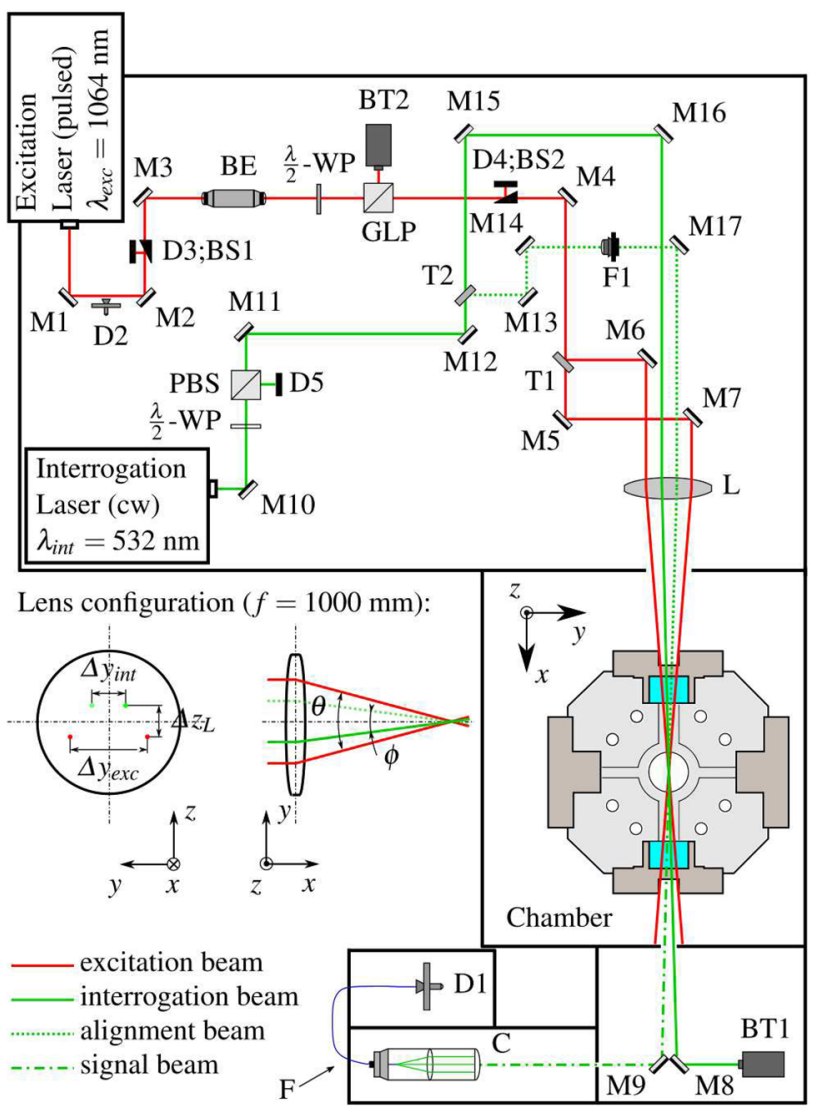

Fig. 3 Optical setup of the LITA system. BE: beam expander; BS: beam sampler; BT: beam trap; C: coupler; D: detector (D1: Avalanche detector; D2: photo diode; D3, D4: pyroelectric sensor; D5: thermal sensor); F: fibre; F1: neutral density filter wheel with orifice; GLP: Glan-Laser polarizer; L: lens; M: mirror; PBS: polarized beam splitter; T: beam splitter; WP: wave plate

all beams into the measurement volume. With an excitation beam distance of $\Delta y_{\text {exc }} \approx 36 \mathrm{~mm}$, the crossing angle yields $\Theta \approx 1^{\circ}$. Based on the laser specifications, the Gaussian halfwidth of the excitation beams in the focal point is estimated to be $\omega_{t h}=312 \mu \mathrm{m}$. Due to the Gaussian beam profile and the beam arrangement the optical measurement volume is an ellipsoid elongated in $x$-direction. Using the modelling proposed by Schlamp et al. (1999), the size of the inference pattern is estimated to be approximately $8.6 \mathrm{~mm}$ in length and $312 \mu \mathrm{m}$ in diameter. This optical interference pattern has a Gaussian intensity profile with a grid spacing $\Lambda$ modulated in $y$-direction, see Siegman (1977). In this context, it is crucial to mention that the direction of propagation of the acoustic waves is normal to the beam direction. Hence, the extension of the effective measurement volume in $x$-direction is smaller than the length of the elliptical interference pattern. The spatial resolution in $x$-direction is, therefore, higher than the optical interference pattern suggests. Evaluating the speed of sound radial profile data provided by Baab et al. (2018) together with the provided shadowgram, we 
estimate the spatial resolution in beam direction to be less than the jet diameter at the measurement location. This leads to a spatial resolution in the present report to of approximately $312 \mu \mathrm{m}$ in diameter and less than $2 \mathrm{~mm}$ in length in the $x$-direction. An avalanche detector (D1; Thorlabs APD110) serves for detection of the scattered signal beam. The latter is previous spatially and spectrally filtered using a coupler and single-mode/multi-mode fibres. The detectors voltage signal is logged with $20 \mathrm{GS} / \mathrm{s}$ by a $1 \mathrm{GHz}$ bandwidth digital oscilloscope (LeCroy, Waverunner 610Zi).

\subsubsection{Post-processing}

The simplest and most common approach to extract speed of sound from a LITA signal is a direct Fourier transformation (DFT). It is imperative to mention that the speed of sound data is directly obtained from the frequency domain of the temporal LITA signal, involving only the geometrical parameters of the optical arrangement. No equation of state or modelling assumptions are necessary at this point. Using the theoretical considerations by Hemmerling and Kozlov (1999), the speed of sound $c_{s}$ of the probed fluid can be estimated as follows:

$c_{s}=\frac{v \Lambda}{j}$.

The dominating frequency of the LITA signal is hereby denoted by $v$. The constant $j$ indicates if the fluid shows resonant behaviour at the wavelength of the excitation beam. In case of non-resonant fluid behaviour $j=2$, whereas in case of resonant fluid behaviour, $j=1$. The grid spacing $\Lambda$ of the optical interference pattern is a calibration parameter for the optical setup. Without a mixing model, thermometry can only be performed at known gas composition and pressure, using a suitable model for the speed of sound. However, the temperature is then only indirectly determined by applying a suitable model for the fluid under consideration. This can be challenging in supercritical or high-pressure states.

Using an analytical approach for finite beam sizes for the evaluation of LITA signals proposed by Schlamp et al. (1999), it should be possible to extract the speed of sound, the acoustic damping rate, as well as the thermal diffusivity from the shape of the LITA signal. In the following, we will summarize the essential parts of the mathematical derivation necessary for this study, as proposed by Schlamp et al. (1999) and Cummings et al. (1995). The used assumptions are categorized and listed in the appendix of this work. As discussed in more detail by Stampanoni-Panariello et al. (2005a), the temporal shape of the excitation laser pulse is estimated using a $\delta$-function at $t_{0}$. The model suggested by Schlamp et al. (1999) can be simplified using two key assumptions proposed by Cummings et al. (1995), namely the limit of fast thermalization and negligible damping over a wave period. Correspondingly, the amplitudes of the acoustic waves $A_{P 1, P 2}$ and the amplitudes of the thermal grating $A_{T}$ in the upcoming modelling equation (13) of the LITA signal simplify to the expressions in equation (9). The real part of $A_{P 1, P 2}$ indicates the influence of thermalization or STBS on the damping oscillation of the LITA signal, while the imaginary part expresses the electrostrictive contribution or SBS. Consequentially, $A_{T}$ represent the weight of thermalization on the signal damping:

$$
\begin{aligned}
A_{P 1, P 2} & =1 / 2 U_{\Theta} \pm i / 2 U_{e P} \\
A_{T} & =-1 U_{\Theta} .
\end{aligned}
$$

The quantities $U_{\Theta}$ and $U_{e P}$ denote the approximate modulation depth of thermalization and electrostriction gratings, respectively (Cummings et al. 1995), which are used as fitting constants. Note that we further assume instantaneous release of absorbed laser radiation into heat, as proposed by Stampanoni-Panariello et al. (2005a). In case of resonant fluid behaviour, both thermalization and electrostriction gratings must be considered. On the other hand, when non-resonant fluid behaviour is expected, the grid generation process is purely electrostrictive. Therefore, the thermal modulation depth $U_{\Theta}$ is negligible leading to a further simplified model. Considering small beam crossing angles and negligible bulk flow velocities, parameters related to the damping of oscillations $\Sigma_{P 1, P 2}$ and the damping parameter $\Sigma_{T}$ can be expressed as given in Eq. (10) referred in Schlamp et al. (1999). Note that, due to negligible bulk flow velocities in the chamber only, beam misalignment in horizontal $y$-direction $\bar{\eta}$ has an effect on the time history of the LITA signal (Schlamp et al. 1999). Hence, after careful beam alignment through the quartz windows before each measurement resulting in a maximized signal, all other possible misalignments are neglected:

$$
\begin{aligned}
\Sigma_{P 1, P 2}= & \exp \left\{-\Gamma q^{2}\left(t-t_{0}\right)-\frac{2\left[\bar{\eta} \pm c_{s}\left(t-t_{0}\right)\right]^{2}}{\omega^{2}+2 \sigma^{2}}\right\} \\
& \exp \left\{ \pm i q c_{s}\left(t-t_{0}\right)\right\} \\
\Sigma_{T}= & \exp \left\{-D_{T} q^{2}\left(t-t_{0}\right)-\frac{2 \bar{\eta}^{2}}{\omega^{2}+2 \sigma^{2}}\right\} .
\end{aligned}
$$

$D_{T}$ denotes the thermal diffusivity, $\Gamma$ the acoustic damping rate, $t$ the time, $t_{0}$ the time of the laser pulse, $\omega$, and $\sigma$ is the Gaussian half-width of the excitation and interrogation beam in the focal point, respectively. The magnitude of the grating wave vector $q$ depends on the grid spacing $\Lambda$, which is a function of the crossing angle $\Theta$ of the excitation beams and the wavelength of the excitation pulse $\lambda_{e x c}$, see Eq. (11) and (12) referred in Stampanoni-Panariello et al. (2005a): 
$q=\frac{2 \pi}{\Lambda}$

$\Lambda=\frac{\lambda_{e x c}}{2 \sin (\Theta / 2)}$.

Using the simplifications explained above and summarized in the appendix, the time-dependent diffraction efficiency $\Psi(t)$ of a detected LITA signal can be expressed as:

$$
\begin{aligned}
\Psi(t) \propto & \exp \left\{-\frac{8 \sigma^{2}}{\omega^{2}\left(\omega^{2}+2 \sigma^{2}\right)}\left(\frac{c_{s}\left(t-t_{0}\right)}{2}\right)^{2}\right\} \\
& \left\{\left(P_{1}+P_{2}\right) T^{*}+\left(P_{1}^{*}+P_{2}^{*}\right) T\right\} \\
& +\exp \left\{-\frac{8 \sigma^{2}}{\omega^{2}\left(\omega^{2}+2 \sigma^{2}\right)}\left(c_{s}\left(t-t_{0}\right)\right)^{2}\right\} \\
& \left(P_{1} P_{2}^{*}+P_{1}^{*} P_{2}\right)+\left(P_{1} P_{1}^{*}+P_{2} P_{2}^{*}+T T^{*}\right) .
\end{aligned}
$$

where the parameters $P_{1}$, T, etc. are calculated using $P_{1}=A_{P 1} \Sigma_{P 1}, T=A_{T} \Sigma_{T}$, etc., and ${ }^{*}$ denotes the complex conjugate. Based on a curve fitting of a LITA signal using modelling Eq. (13), an estimation of the thermodynamic variables $c_{s}, D_{T}$ and $\Gamma$ is possible. Based on these transport properties, experimental investigations using LITA in the vicinity of the critical point and in the vicinity of the Widom line of a pure fluid disclose the possibility to study transitions between the supercritical fluid states depicted in Fig. 1 on a macroscopic level. Additionally, given a suitable thermodynamic model for these parameters, the knowledge of local transport properties enables us, as shown in Eq. (4), to extract local mixture quantities with a spatial resolution $\mathcal{O}\left(10^{-1} \mathrm{~mm}\right)$ in diameter and $\mathcal{O}\left(10^{0} \mathrm{~mm}\right)$ in length.

\section{Results}

Extraction of transport properties from LITA signals, using the approach explained above, requires a thorough calibration of the optical setup as well as a validation of the acquired transport properties. Both calibration and validation are presented in the following section. The uncertainty analysis of the operating conditions as well as the Fourier analysis of the LITA signal, the calibration, and validation of the grid spacing $\Lambda$ is performed according to the Guide to the expression of uncertainty in measurement by the Joint Committee for Guides in Metrology (2008). For values taken from a database, uncertainties are acquired using sequential perturbation as presented by Moffat (1988).

Since the purpose of this study is the feasibility to extract transport properties from LITA signals, the presented uncertainties of the acoustic damping rates gained by the curve fitting algorithm are based on the confidence interval computed by the algorithm. These confidence intervals are estimated using the inverse $R$ factor from the $Q R$ decomposition of the Jacobian, the degrees of freedom, as well as the root-mean-squared error. Hence, the uncertainties of the acoustic damping rates are only a representation of the statistical error margin of the curve fitting at this point and do not take the uncertainties of the fitted data and the input parameters into account. The uncertainties of speed of sound data extracted using curve fitting are estimated based on the results for the DFT analysis. All uncertainties are presented within a confidence interval of $95 \%$.

\subsection{Calibration of optical setup}

Modelling of LITA signals requires a deep understanding of non-linear optical processes, and phonon-photon as well as thermon-photon interaction inherent to the LITA measurement technique (Cummings et al. 1995). Additionally, Eq. (13) is highly dependent on the beam waist of the excitation beam $\omega$ as well as the magnitude of the grating vector $q$, which depends on the spacing of the optical grid $\Lambda$. Both values are highly vulnerable to distortions due to turbulence and convective transport processes if they occur in a similar time scale. However, averaging the signal over a high number of laser pulses smears the signal and minimizes the effect of shot to shot variations in turbulence and convective processes as well as laser noise, jitter, and drift. An independent study to quantify these effects, however, is highly complex. Using Eq. (8) and/or (13) to measure speed of sound, acoustic damping rate or thermal diffusivity requires, therefore, a careful and thorough calibration of the optical measurement volume, specifically the spacing of the optical grid $\Lambda$ and the Gaussian half-width $\omega$ of the excitation beam.

The calibration is done in well-known quiescent conditions. When assuming non-resonant behaviour, the grid spacing of the optical measurement volume can be characterized by the excitation wavelength $\lambda_{\text {exc }}$ :

$\Lambda\left(\Delta y_{\text {exc }}, f_{\text {exc }}, \lambda_{\text {exc }}\right)=2 \frac{c_{s}}{v}$.

A beam profiling camera (DataRay) is used for beam alignment and to measure the beam arrangement in the foci of the excitation and the interrogation beams at atmospheric conditions. Using the collected geometrical data, the grid spacing is estimated to be $\Lambda_{B P}=29.6 \pm 7 \mu \mathrm{m}$ with a beam distance of $\Delta y_{e x c, B P}=36.9 \pm 3.8 \mathrm{~mm}$. Despite the agreement of the geometrical calibration using the beam profiling camera and the known parameters of the used optical setup, the measurement uncertainties of the calibration are unacceptable. Therefore, a new calibration procedure has been developed to calibrate the optical setup. The grid spacing is estimated for conditions up to $700 \mathrm{~K}$ and $8 \mathrm{MPa}$ in non-resonant fluids, 
namely nitrogen and argon. Using the dependencies of the grid spacing $\Lambda$ in Eq. (14) together with the speed of sound $c_{s}$ extracted from Lemmon et al. (2018), we are able to calculate a mean grid spacing $\Lambda$. It is important to emphasise that the grid spacing is, as shown by Li et al. (2002), solely dependent on the geometrical and optical parameters of the optical setup $\left(f, \Delta y_{e x c}, \lambda_{e x c}\right)$. Hence, it is independent of the refractive index of the probed environment. The dominating frequency of the LITA signal $v$ is estimated using a DFT together with a von Hann window and a band-pass filter. This ensures the correct extraction of the frequencies even for noisy signals in gas, gas-like, and compressed liquid states. Note that, for each condition, the acquired frequencies are averaged over at least 5000 samples. The calibration procedure yields a grid spacing of the measurement volume of $\Lambda_{\text {cal }}=29.33 \pm 0.14 \mu \mathrm{m}$. Note that the measurement uncertainties using the new calibration procedure are more than one order of magnitude below the calibration using the beam profiling camera.

Due to the high spatial resolution required for investigations in the wake of free-falling evaporation droplets and jet disintegration, the optical arrangement uses focused beams with a Gaussian beam profile. Hence, calibration of the beam waist is crucial for the correct modelling of the LITA signal and robust extraction of the acoustic damping rate as well as the thermal diffusivity. Calibration is performed using LITA signals with different operating conditions, as listed in Table 1. All signals are averaged over $n_{\text {LITA }}$ laser shots. To acquire the Gaussian half-width $\omega$, the experimentally detected and averaged LITA signals presented in Table 1 are curve fitted using the simplified model by Schlamp et al. (1999) expressed by Eq. (13), which is presented in Sect. 3.2.2. A robust non-linear least-absolute fit using the Levenberg-Marquardt algorithm is utilized by using the non-linear fit in Matlab (MathWorks) with the robust option Least-Absolute Residuals (LAR). This method optimizes the fit by minimizing the absolute differences of the residual rather than the squared differences. We have chosen this option instead of an approach using bisquare weights, since signals averaged over more than 5000 laser shots are fitted, which leads to few outliers. The Gaussian beam width $\omega$, the modulation depths $U_{e P}, U_{\Theta}$, the beam misalignment $\bar{\eta}$, the speed of sound $c_{s}$, and the temporal offset $t_{0}$ are hereby output parameters, whereas the remaining parameters are input parameters, which were kept constant during the curve fitting. The grid spacing is set to the calibrated value and the Gaussian half-width of the interrogation beam to the value measured by beam profile camera $\sigma_{B P}$. Transport properties are estimated using Lemmon et al. (2018) for thermal diffusivity and shear viscosity. Acoustic damping rates are assessed by the model proposed by Li et al. (2002), see Eq. (3). The curve fitting of the last case listed in Table 1 is exemplarily depicted in Fig. 4.

Assuming an exponential dependence with negative exponent of pulse energy and Gaussian half beam width $\omega$ of the excitation beam, $\omega$ can be expressed as:

$\omega_{c a l}=\omega_{t h}-\left(\omega_{t h}-\omega_{B P}\right) \exp \left\{-\alpha_{e x c} E_{e x c}\right\}$,

where $\alpha_{e x c}$ is the calibration constant, $\omega_{B P}=190 \mu \mathrm{m}$ is the Gaussian half-width measured with the beam profiling camera, and $\omega_{t h}=312 \mu \mathrm{m}$ is the Gaussian half-width estimated using the laser specifications. Note that for high pulse energies $\omega$ approaches $\omega_{t h}$, whereas for low pulse energies $\omega$ can be approximated by the investigations using the beam profiling camera. A robust non-linear least square fit using the Levenberg-Marquardt algorithm with bisquare weights is used to acquire $\alpha_{e x c}$.
Table 1 Overview of the operating conditions, input parameters, and results of the calibration of the Gaussian half-width of the excitation beam $\omega$; operating conditions: $T_{c h}$ : fluid temperature; $p_{c h}$ : fluid pressure; $n_{\text {LITA }}:$ number of laser shots used for averaging; $E_{\text {exc }}:$ pulse energy of excitation beams; $P_{\text {int }}$ : power of interrogation beam; SM: single-mode fibre with diameter of $4 \mu \mathrm{m}$; MM: multi-mode fibre with diameter of $25 \mu \mathrm{m}$; curve fitting input parameters: calibrated grid spacing $\Lambda_{\text {cal }}=29.33 \mu \mathrm{m}$; measured Gaussian half-width of the interrogation beam $\sigma_{B P}=192 \mu \mathrm{m} ; D_{T, \mathrm{NIST}}$ : thermal diffusivity; $\Gamma_{m, \mathrm{NIST}}$ : pressure corrected acoustic damping rates; curve fitting results: $c_{s, \text { LITA }}:$ speed of sound; $\omega_{\text {LITA }}:$ Gaussian half-width of the excitation beam

\begin{tabular}{|c|c|c|c|c|c|c|c|c|c|c|}
\hline Gas & $p_{c h}$ in $\mathrm{MPa}$ & $T_{c h}$ in $\mathrm{K}$ & $E_{e x c}$ in $\mathrm{mJ}$ & $P_{i n t}$ in $\mathrm{W}$ & Fibre & $n_{\text {LITA }}$ & $c_{s, \mathrm{LITA}}$ in $\mathrm{ms}^{-1}$ & $\begin{array}{l}\Gamma_{m, \mathrm{NIST}} \text { in } \\
\mathrm{mm}^{2} \mathrm{~s}^{-1}\end{array}$ & $\begin{array}{l}D_{T, \mathrm{NIST}} \text { in } \\
\mathrm{mm}^{2} \mathrm{~s}^{-1}\end{array}$ & $\omega_{\mathrm{LITA}}$ in $\mu \mathrm{m}$ \\
\hline $\mathrm{N}_{2}$ & $2.0 \pm 0.1$ & $295.6 \pm 1.8$ & 40.0 & 8.0 & SM & 6057 & $356 \pm 4$ & 3.25 & 1.08 & $277 \pm 2$ \\
\hline $\mathrm{Ar}$ & $2.0 \pm 0.1$ & $294.7 \pm 0.6$ & 22.5 & 8.0 & SM & 9901 & $313 \pm 2$ & 1.37 & 1.01 & $259 \pm 2$ \\
\hline $\mathrm{Ar}$ & $2.0 \pm 0.1$ & $295.3 \pm 0.6$ & 22.5 & 6.0 & SM & 9334 & $317 \pm 2$ & 1.38 & 1.02 & $251 \pm 2$ \\
\hline $\mathrm{Ar}$ & $2.0 \pm 0.1$ & $295.2 \pm 0.6$ & 22.5 & 4.0 & MM & 10713 & $320 \pm 2$ & 1.38 & 1.02 & $241 \pm 2$ \\
\hline $\mathrm{Ar}$ & $2.0 \pm 0.1$ & $293.9 \pm 0.6$ & 27.0 & 4.0 & MM & 5283 & $324 \pm 2$ & 1.37 & 1.01 & $301 \pm 1$ \\
\hline $\mathrm{Ar}$ & $0.5 \pm 0.1$ & $295.4 \pm 0.6$ & 32.4 & 5.0 & MM & 8650 & $316 \pm 2$ & 3.78 & 4.13 & $209 \pm 2$ \\
\hline $\mathrm{Ar}$ & $1.0 \pm 0.1$ & $295.2 \pm 0.6$ & 32.4 & 4.0 & MM & 10100 & $315 \pm 2$ & 2.17 & 2.05 & $222 \pm 2$ \\
\hline $\mathrm{Ar}$ & $2.0 \pm 0.1$ & $295.2 \pm 0.6$ & 32.4 & 1.5 & $\mathrm{MM}$ & 10602 & $316 \pm 2$ & 1.38 & 1.02 & $216 \pm 1$ \\
\hline
\end{tabular}




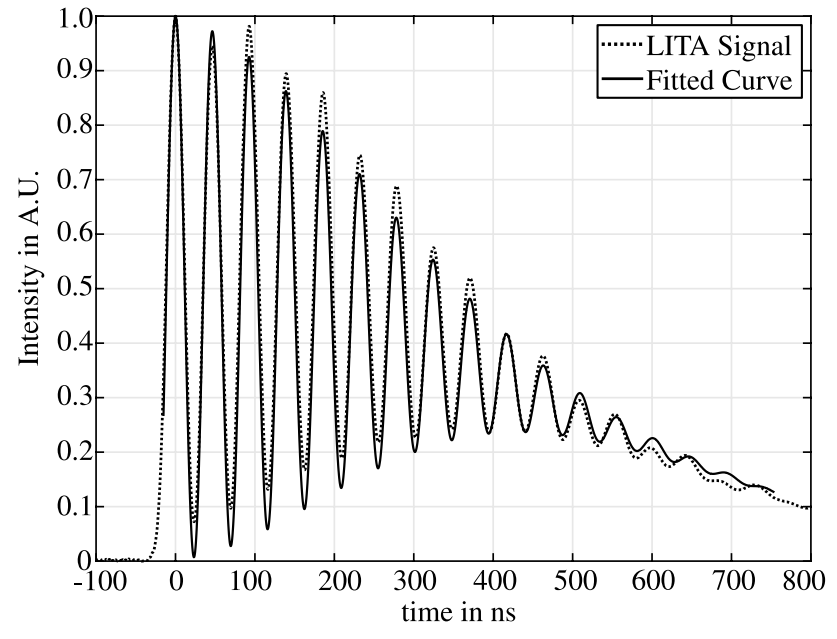

Fig. 4 Measured LITA signal with non-resonant fluid behaviour in pure argon with curve fitting result used for calibration. The signal is averaged over 10602 laser shots. Operating conditions: $\quad p_{c h}=2 \pm 0.1 \mathrm{MPa} ; \quad T_{c h}=295.2 \pm 0.6 \mathrm{~K} ; \quad E_{e x c}=32.4 \mathrm{~mJ}$; $P_{\text {int }}=1.5 \mathrm{~W}$; Curve fitting input parameters: $\Lambda=\Lambda_{\text {cal }}=29.33 \mu \mathrm{m}$; $\sigma=\sigma_{B P}=192 \mu \mathrm{m} ; \quad \Gamma=\Gamma_{m, \mathrm{NIST}}=1.38 \mathrm{~mm}^{2} \mathrm{~s}^{-1}$; $D_{T}=D_{T, \mathrm{NIST}}=1.02 \mathrm{~mm}^{2} \mathrm{~s}^{-1}$; $\quad$ Curve fitting results: $\omega_{\text {LITA }}=216 \pm 1 \mu \mathrm{m} ; c_{s, \text { LITA }}=316 \pm 2 \mathrm{~m} \mathrm{~s}^{-1}$

\subsection{Validation and analysis of measurement uncertainties}

Due to the availability of speed of sound data at a wide range of pressure and temperature, the validation of the optical grid spacing $\Lambda$ using Eq. (8) is possible in the whole operating range. However, since acoustic damping rates depend on both bulk and shear viscosities, available data for hightemperature and high-pressure environments are rare. Hence, validation is performed in the following section using only acoustic damping rates of argon at room temperature.

\subsubsection{Experimental speed of sound data}

Figure 5 depicts the validation of the calibration process using the grid spacing to characterize the measurement volume. The speed of sound was calculated by Eq. (8), where the non-resonant frequency was estimated using a DFT together with a von Hann window and a band-pass filter. A relative distribution is shown in Fig. 6. For clarity, we omit the distinction between the different non-resonant fluids argon and nitrogen in the distribution. Both distributions show a Gaussian shaped distribution. For non-resonant cases, the skewness of the distribution is -0.3 with a kurtosis of 3.3. In case of resonant fluid behaviour, skewness is -1 with a kurtosis of 3.7.

Validation of the calibration process shows good agreement between the non-resonant measurements of nitrogen and argon, and theoretical values extracted from Lemmon et al. (2018) (NIST database). The relative measurement uncertainty of the acquired speed of sound for all investigated fluids is below $2 \%$. For argon and carbon dioxide, the uncertainties of measurement and NIST database are in the same order of magnitude, which indicates the good
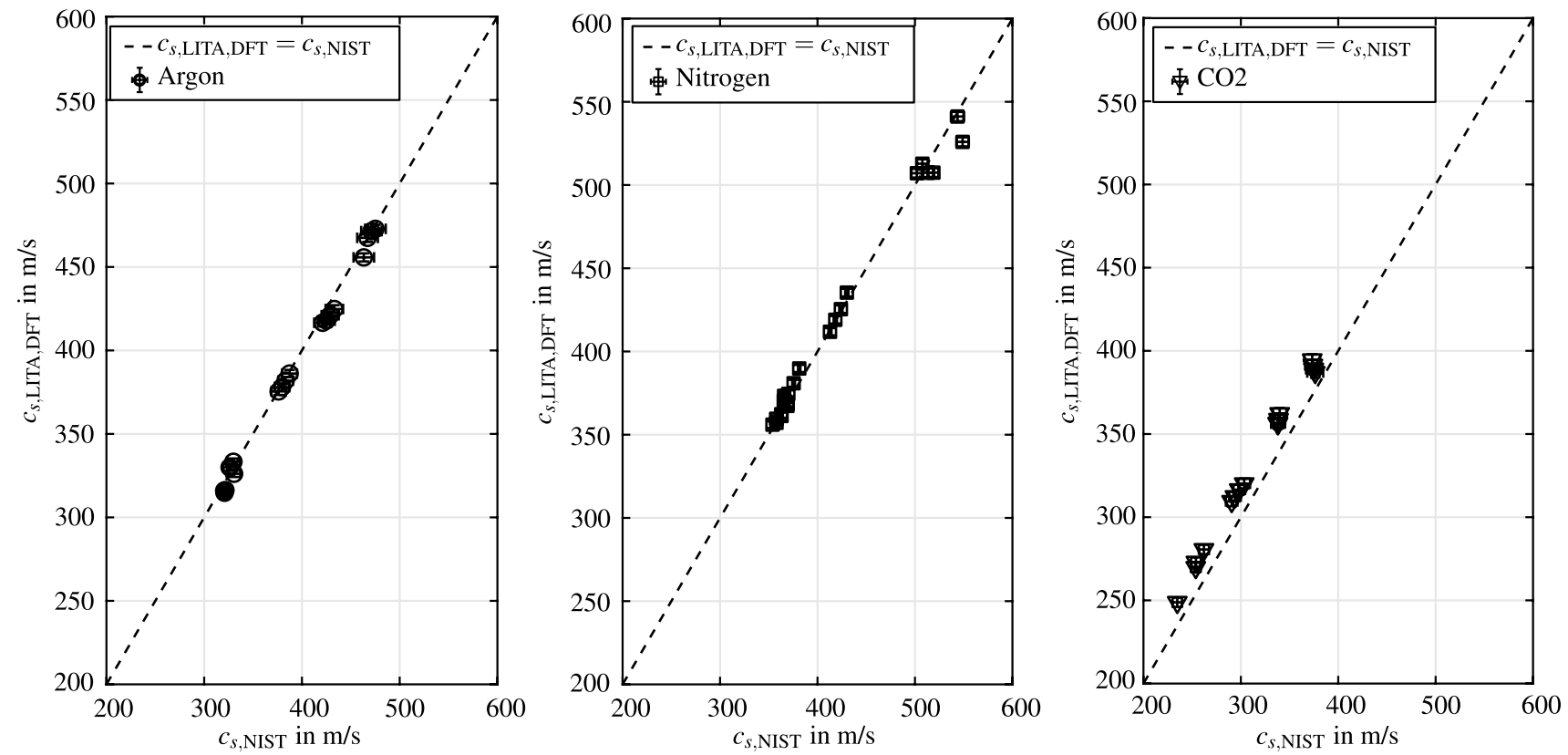

Fig. 5 Absolute comparison of speed of sound data for $p_{c h}=2$ to $8 \mathrm{MPa}$ and temperatures up to $T_{c h}=700 \mathrm{~K}$ (nitrogen) and $p_{c h}=0.5$ to $8 \mathrm{MPa}$ and temperatures up to $T_{c h}=600 \mathrm{~K}$ (argon and carbon dioxide) using a grid spacing of $\Lambda=\Lambda_{\text {cal }}=29.33 \pm 0.14 \mu \mathrm{m}$. The speed of sound is calculated using a DFT with Eq. (8). Thermodynamic data for validation are taken from Lemmon et al. (2018) 


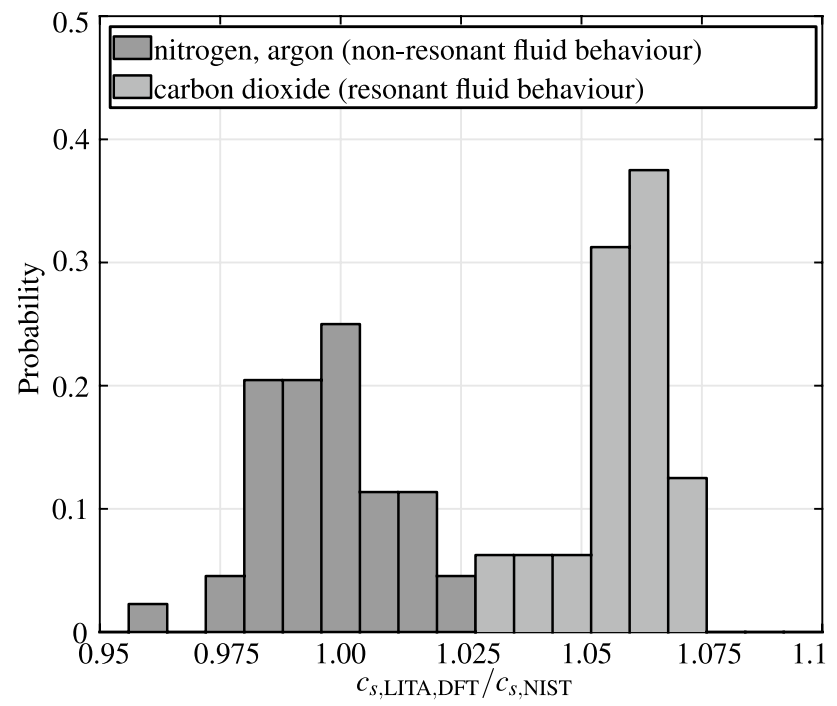

Fig. 6 Relative distribution of the comparison of speed of sound data for $p_{c h}=2$ to $8 \mathrm{MPa}$ and temperatures up to $T_{c h}=700 \mathrm{~K}$ (nitrogen) and $p_{c h}=0.5$ to $8 \mathrm{MPa}$ and temperatures up to $T_{c h}=600 \mathrm{~K}$ (argon and carbon dioxide) using a grid spacing of $\Lambda=\Lambda_{\text {cal }}=29.33 \pm 0.14 \mu \mathrm{m}$. The speed of sound is calculated using a DFT with Eq. (8). Thermodynamic data for validation are taken from Lemmon et al. (2018). For carbon dioxide, the skewness of the distributions is -1 with a kurtosis of 3.7 ; for nitrogen, argon skewness is acquired to be -0.3 with a kurtosis of 3.3

precision of the LITA setup. However, the distribution in Fig. 6 shows a width of approximately $3 \%$ for non-resonant fluids argon and nitrogen. These differences result from unavoidable misalignments of the excitation beams as well as beam steering effects due to the high-temperature and highpressure environment.

Measurements with resonant fluid behaviour observed for carbon dioxide, however, show a consistent deviation, resulting in an offset between the measurements and theoretical values taken from Lemmon et al. (2018). In addition to the observed offset in validation for carbon dioxide at temperatures up to $600 \mathrm{~K}$ and pressures up to $8 \mathrm{MPa}$, a resonant fluid behaviour is observed. However, based on the absorption cross-section of carbon dioxide, resonant fluid behaviour can be a priori excluded. One and the most probable explanation for these resonant behaviours is residual moisture in the experimental setup. With the absorption cross-section of water at the excitation wavelength, residual moisture would cause resonant fluid behaviour as has already been observed by Cummings (1995). The constant offset even for low mole fractions of water also demonstrates quite nicely the sensitivity of the LITA signal on the concentration of a mixture. This sensitivity is essential to extract the mixing temperature as well as the mole fraction using Eq. (13) together with the relations shown in (4).

To ensure the robustness of the optical setup, additional investigations in carbon dioxide are conducted. The objective of these experiments is twofold. First, experimental investigation in an open loop setup with carbon dioxide after drying the experimental setup using elevated temperature and vacuum shows no influence of residual moisture on the LITA signal and the calibration procedure. Hence, the origin of residual moisture is most likely due to purity of the used carbon dioxide. Second, the authors propose an intensity study, in which the energy of the excitation laser is systematically varied. This could shed some light on two other possible explanations for the observed resonant fluid behaviour, namely spontaneous Raman scattering or an optical breakdown of carbon dioxide. The authors hypothesize that due to the high pulse energy of up to $32 \mathrm{~mJ}$ used for LITA measurements in carbon dioxide an optical breakdown of the fluid might occur, which would cause changes in the fluid properties. This would affect the formation of the density grating and, therefore, the measured LITA signal. A detailed description can be found in the work of StampanoniPanariello et al. (2005a). Additionally, the dependencies of the intensity of the detectable LITA signal on the excitation beam pulse energy are different for non-resonant LITA and spontaneous Raman scattering. Note that non-resonant, purely electrostrictive LITA signals are caused by stimulated Brillouin scattering (SBS), whereas resonant, LITA signals are caused by the combination of SBS, stimulated thermal Brillouin scattering (STBS), and/or stimulated thermal Rayleigh scattering (STRS). With reference to the theoretical work by Stampanoni-Panariello et al. (2005a), the signal intensity of the detectable LITA signal for both resonant and non-resonant fluid behaviour shows a quadratic dependence on the pulse energies of the excitation beams. Spontaneous Raman scattering experiences, however, a linear dependence on the incident intensity, see Powers (2013). Starting from low-energy pulses, a systematic increase of the pulse energy should, therefore, indicate when an optical breakdown of carbon dioxide occurs and if spontaneous Raman scattering is the cause of the resonant fluid behaviour, by favouring one of the mentioned effects over the other.

\subsubsection{Comparison of experimental and theoretical LITA signals}

Acoustic damping rate, speed of sound, and thermal diffusivity are gained using curve fitting of experimentally detected, averaged LITA signals with the simplified model by Schlamp et al. (1999) expressed by Eq. (13) presented in Sect. 3.2.2.

Similar to the curve fit in the calibration procedure, a robust non-linear least-absolute curve fit is used. The latter utilizes a Levenberg-Marquardt algorithm. Besides the desired speed of sound $c_{s}$, acoustic damping rate $\Gamma$, thermal diffusivity $D_{T}$, the modulation depths $U_{e P}, U_{\Theta}$, the beam misalignment $\bar{\eta}$, and the temporal offset $t_{0}$, all parameters 
are input parameters, which were held constant during the curve fitting. The grid spacing $\Lambda$ and Gaussian half-width of the excitation beam $\omega$ are set to the calibrated value. The Gaussian half-width of the interrogation beam $\sigma_{B P}$ is held constant at the value measured by the beam profile camera. A curve fit for a LITA signal in pure argon at a pressure of $4 \mathrm{MPa}$ and a temperature of $295 \mathrm{~K}$ is shown in Fig. 7. The signal is averaged over 10991 shots. Curve fitting yields $c_{s, \text { LITA }}=319 \pm 2 \mathrm{~m} \mathrm{~s}^{-1}$ and $\Gamma_{\text {LITA }}=1.2 \pm 0.1 \mathrm{~mm}^{2} \mathrm{~s}^{-1}$, which compared to theoretical estimations using Eq. (3) together with the NIST database by Lemmon et al. (2018) $\left(c_{s, \mathrm{NIST}}=324 \mathrm{~m} \mathrm{~s}^{-1}\right.$ and $\left.\Gamma_{m, \mathrm{NIST}}=1.0 \mathrm{~mm}^{2} \mathrm{~s}^{-1}\right)$ show good agreement. The feasibility to extract acoustic damping rates in a high-pressure, high-temperature environment for a resonant fluid is presented in Fig. 8. A carbon dioxide atmosphere with residual moisture at pressure of $8 \mathrm{MPa}$ and a temperature of $502.5 \mathrm{~K}$ is shown. Note that, due to the low modulation depth of the thermal grating compared to the electrostrictive grating $U_{\Theta, \text { LITA }} / U_{e P, \text { LITA }}<0.08$, the fitted thermal diffusivity does not reveal physically realistic results.

Figure 9 depicts acoustic damping rate ratios $\Gamma_{\mathrm{LITA}} / \Gamma_{c, \mathrm{NIST}}$ for pure argon at room temperature for various pressures up to $8 \mathrm{MPa}$. Values are compared to the experimental and theoretical investigations by Li et al. (2002). For pressures up to $4 \mathrm{MPa}$, our experimental investigation shows good consensus with data by Li et al. (2002). The points

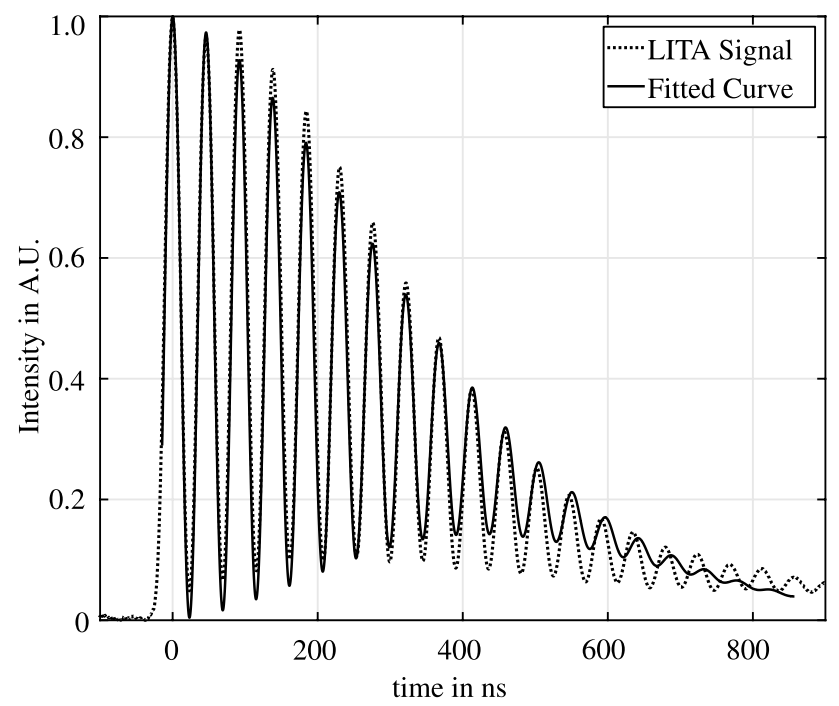

Fig. 7 Measured LITA signal with non-resonant fluid behaviour in pure argon with curve fitting result used to acquire transport properties. The signal is averaged over 10991 laser shots. Operating conditions: $p_{c h}=4 \pm 0.1 \mathrm{MPa} ; T_{c h}=295.0 \pm 0.5 \mathrm{~K} ; \quad E_{e x c}=22.5 \mathrm{~mJ}$; $P_{\text {int }}=2.5 \mathrm{~W}$; curve fitting input parameters: $\Lambda=\Lambda_{\text {cal }}=29.33 \mu \mathrm{m}$; $\sigma=\sigma_{B P}=192 \mu \mathrm{m} ; \quad \omega=\omega_{c a l}=238 \mu \mathrm{m} ; \quad$ curve fitting results: $c_{s, \mathrm{LITA}}=319 \pm 2 \mathrm{~m} \mathrm{~s}^{-1} ; \Gamma_{\text {LITA }}=1.2 \pm 0.1 \mathrm{~mm}^{2} \mathrm{~s}^{-1}$; theoretical estimations using Eq. (3) together with the NIST database by Lemmon et al. (2018) give: $c_{s, \mathrm{NIST}}=324 \mathrm{~m} \mathrm{~s}^{-1}$ and $\Gamma_{m, \mathrm{NIST}}=1.0 \mathrm{~mm}^{2} \mathrm{~s}^{-1}$

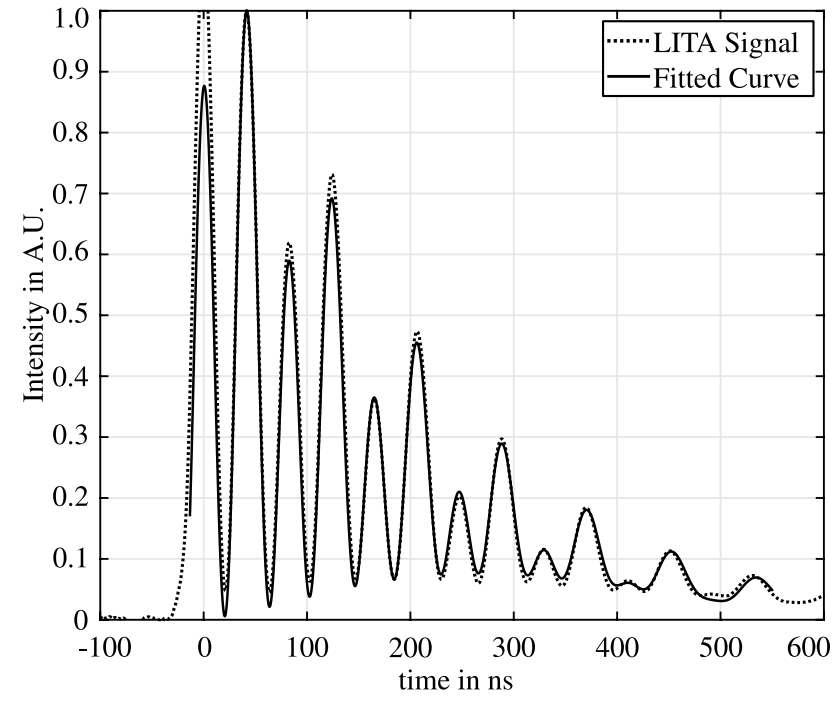

Fig. 8 Measured LITA signal with resonant fluid behaviour in carbon dioxide with curve fitting result used to acquire transport properties. The signal is averaged over 11030 laser shots. Operating conditions: $p_{c h}=8 \pm 0.1 \mathrm{MPa} ; T_{c h}=502.5 \pm 4.9 \mathrm{~K} ; \quad E_{\text {exc }}=18 \mathrm{~mJ}$; $P_{\text {int }}=1 \mathrm{~W}$; curve fitting input parameters: $\Lambda=\Lambda_{\text {cal }}=29.33 \mu \mathrm{m}$; $\sigma=\sigma_{B P}=192 \mu \mathrm{m} ; \quad \omega=\omega_{\text {cal }}=230 \mu \mathrm{m} ; \quad$ curve fitting results: $c_{s, \mathrm{LITA}}=356 \pm 2 \mathrm{~m} \mathrm{~s}^{-1} ; \Gamma_{\mathrm{LITA}}=41 \pm 1 \mathrm{~mm}^{2} \mathrm{~s}^{-1}$

at same temperatures and pressures indicate experiments with similar operating conditions, which are investigated on different days. The deviation between those points is most likely caused by beam steering effects of the excitation beams due to staining of the quartz windows resulting from

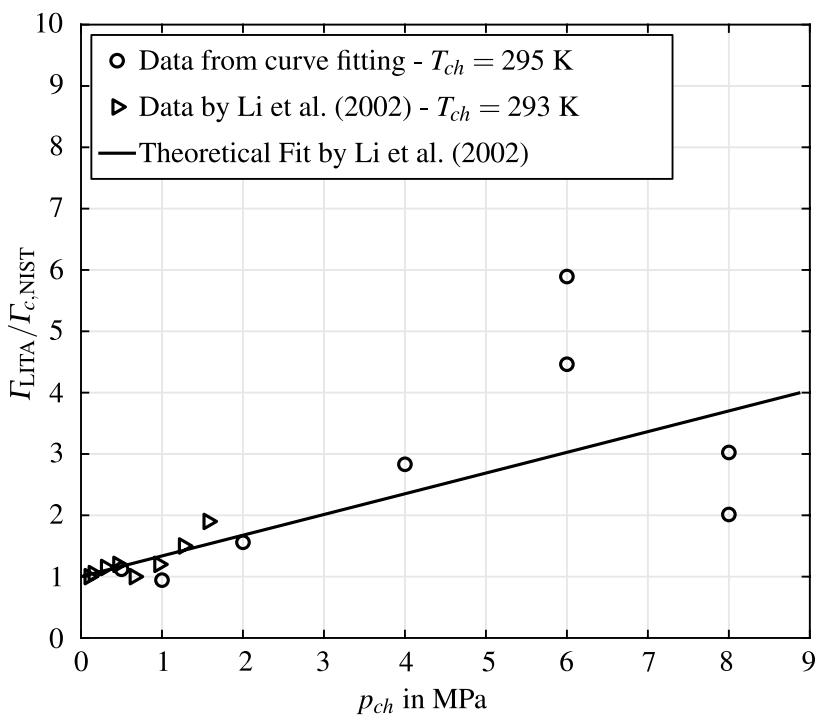

Fig. 9 Acoustic damping rate ratio $\Gamma_{\mathrm{LITA}} / \Gamma_{c, \mathrm{NIST}}$ over chamber pressure $p_{c h}$ for pure argon at room temperature. Classical acoustic damping rates are estimated using NIST database by Lemmon et al. (2018). Experimental and theoretical data are taken from Li et al. (2002) 
a slightly excessive pulse energy. These stains could change the Gaussian beam width of the excitation beams without changing the beam crossing angle. Acoustic damping rate ratios at higher pressure do not show a linear dependence on pressure. Considering the critical pressure of argon $p_{c}=4.9 \mathrm{MPa}$, deviations are expected, since shear and bulk viscosity show non-linear behaviour in the vicinity of the critical point as indicated by Meier et al. (2004) and Meier et al. (2005).

The authors hypothesize that the high values of acoustic damping rates in Fig. 9 result from thermodynamic anomalies in the vicinity of the critical point. These anomalies are an exponential increase in shear viscosity above the critical pressure as shown by Meier et al. (2004) and peak values in bulk flow velocities in the vicinity of the critical pressure as has been simulated for Lennard-Jones fluids by Meier et al. (2005). We further emphasise that bulk viscosities are neglected in the theoretical fit by Li et al. (2002), and that the fit is only validated for pressures up to $2.53 \mathrm{MPa}$. The comparison in Fig. 9 indicates the capability of spatially high-resolved LITA measurements to extract speed of sound and acoustic damping rates in fluids. However, for a more concise calibration, theoretical or experimental data of acoustic damping rate at high pressure and temperatures are necessary. Hence, further investigations of theoretical approximations and experimental data of bulk viscosities are essential to validate the presented post processing curve fitting algorithm for high pressure.

\section{Conclusion}

In this study, the challenges as well as the potential of laser-induced thermal acoustics for small-scale macroscopic fluid phenomena occurring in jet disintegration or droplet evaporation are presented. By applying LITA with an optical arrangement using focused beams, we can successfully acquire transport properties using an elliptical measurement volume with a spatial resolution of $312 \mu \mathrm{m}$ in diameter and less than $2 \mathrm{~mm}$ in length. The speed of sound is measured in a high-pressure and high-temperature environment for various fluids using LITA together with a direct Fourier analysis. To validate these measurements and access their measurement uncertainties, a comparison with the NIST database by Lemmon et al. (2018) is implemented. Using a confidence interval of $95 \%$, the relative uncertainties for the speed of sound data are within $3 \%$ of the acquired values. Furthermore, acoustic damping rates are acquired by curve fitting experimental LITA signals to a simplified analytical expression based on the model by Schlamp et al. (1999). Validation using pure argon at elevated pressures shows promising results and, hence, confirm the capability of LITA to simultaneously measure transport properties in small-scale fluid phenomena. The importance of these transport properties measured using LITA is twofold. Investigations in pure fluids in the vicinity of their critical point and across their Widom line enable us to study transitions between supercritical fluid states on a macroscopic level. Moreover, by applying suitable models for speed of sound, acoustic damping rates, as well as thermal diffusivities in fluid mixtures, it is possible to determine mixing parameters in macroscopic fluid phenomena on a small scale.

Acknowledgements The authors gratefully acknowledge the financial support by the Deutsche Forschungsgemeinschaft (DFG, German Research Foundation)-Project SFB-TRR 75, Project number 84292822.

Funding Open Access funding enabled and organized by Projekt DEAL. This study was funded by the Deutsche Forschungsgemeinschaft (DFG, German Research Foundation)—Project SFB-TRR 75, Project number 84292822 .

Data availability statement The experimental data are not yet publicly available.

\section{Compliance with ethical standards}

Conflict of interest The authors declare that they have no conflict of interest.

Code availability Calibration, validation, and curve fitting codes are implemented using MATLAB (MathWorks). The code is not publicly available.

Open Access This article is licensed under a Creative Commons Attribution 4.0 International License, which permits use, sharing, adaptation, distribution and reproduction in any medium or format, as long as you give appropriate credit to the original author(s) and the source, provide a link to the Creative Commons licence, and indicate if changes were made. The images or other third party material in this article are included in the article's Creative Commons licence, unless indicated otherwise in a credit line to the material. If material is not included in the article's Creative Commons licence and your intended use is not permitted by statutory regulation or exceeds the permitted use, you will need to obtain permission directly from the copyright holder. To view a copy of this licence, visit http://creativecommons.org/licenses/by/4.0/.

\section{Appendix}

The temporal shape of the excitation laser pulse can be estimated using a $\delta$-function at $t_{0}$. As discussed by Stampanoni-Panariello et al. (2005a), this assumption is valid since the laser pulse length $\tau$ :

- is small compared to the reciprocal of the acoustic frequency $\left(\tau \ll 1 /\left(c_{s} q\right)\right)$; 
- is small compared to the reciprocal of the acoustic decay rates $\left(\tau \ll 1 /\left(q^{2} \Gamma\right)\right)$ and $\left(\tau \ll 1 /\left(q^{2} D_{T}\right)\right)$

The following assumptions are used to derive Eq. (9) in this work from Eqs. (6d), (6e) and (15b) in the work of Schlamp et al. (1999). The assumptions are based on the theoretical considerations by Cummings et al. (1995) and Stampanoni-Panariello et al. (2005a):

- Cummings et al. (1995) proposed that in the limit of fast thermalization $D_{T} \ll 1$, where $D_{T}$ denotes the thermal diffusivity.

- Cummings et al. (1995) proposed that, due to negligible damping over a wave period $\Gamma \ll 1$, where $\Gamma$ denotes the acoustic damping rate.

- Stampanoni-Panariello et al. (2005a) proposed that, in case of instantaneous release of absorbed laser radiation into heat $c_{s} q \ll \gamma_{\Theta}+\gamma_{n \Theta}$, where $c_{s}$ denotes the speed of sound, $q$ denotes the magnitude of the grating wave vector, and $\gamma_{\Theta}$ denotes the rate of excited-state energy decay caused by thermalization (in the work of StampanoniPanariello et al. (2005a), this parameter is denoted as $\lambda$ ) and $\gamma_{n \Theta}$ denotes the rate of excited-state energy decay not caused by thermalization (in the work of StampanoniPanariello et al. (2005a), this parameter is denoted as $\psi$ ).

- Note that, using the above assumptions, Eq. (15b) in the work of Schlamp et al. (1999) yields $A_{D}=0$, which is not shown in Eq. (9) in this work.

Equation (10) in this work is derived from Eqs. (3b), (13b), (14d) in the work of Schlamp et al. (1999) using the following assumptions and simplifications based on the specific conditions of presented experimental investigations:

- Considering the small beam crossing angles in the optical setup, we assume $\cos (\Theta) \approx 1$ and $\cos (\Phi) \approx 1$.

- Due to the low mass flow rates during the investigations and the vanishing flow velocities, bulk flow velocities can be neglected: $v=0$ and $w=0$.

- Due to negligible bulk flow velocities in the chamber only beam misalignment in horizontal $y$-direction, $\bar{\eta}$ has an effect on the time history of the LITA signal (Schlamp et al. 1999). Hence, after careful beam alignment through the quartz windows before each measurement resulting in a maximized signal, the beam misalignment in $z$-direction is neglected $\bar{\zeta}=0$.

- Note that, due to the simplified version of Eq. (9) in this work, $A_{D}=0$; therefore, the value $D=A_{D} \Sigma_{D}=0$ and it is not necessary to calculate $\Sigma_{D}$ in Eq. (14d) from the work of Schlamp et al. (1999).

Following the simplifications and assumptions summarized above and considering only the temporal evolution of the
LITA signal Eq. (17) in the work of Schlamp et al. (1999) can be further simplified to Eq. (13) proposed in this work.

\section{References}

Baab S, Förster FJ, Lamanna G, Weigand B (2016) Speed of sound measurements and mixing characterization of underexpanded fuel jets with supercritical reservoir condition using laser-induced thermal acoustics. Exp Fluids 57(11):3068. https://doi.org/10.1007/ s00348-016-2252-3

Baab S, Steinhausen C, Lamanna G, Weigand B, Förster FJ (2018) A quantitative speed of sound database for multi-component jet mixing at high pressure. Fuel 233:918-925. https://doi.org/10.1016/j. fuel.2017.12.080

Bencivenga F, Cunsolo A, Krisch M, Monaco G, Ruocco G, Sette F (2009) High frequency dynamics in liquids and supercritical fluids: a comparative inelastic X-ray scattering study. J Chem Phys 130(6):064501. https://doi.org/10.1063/1.3073039

Bork B, Preusche A, Weckenmann F, Lamanna G, Dreizler A (2017) Measurement of species concentration and estimation of temperature in the wake of evaporating n-heptane droplets at trans-critical conditions. Proc Combustion Inst 36(2):2433-2440. https://doi. org/10.1016/j.proci.2016.07.037

Crua C, Manin J, Pickett LM (2017) On the transcritical mixing of fuels at diesel engine conditions. Fuel 208:535-548. https://doi. org/10.1016/j.fuel.2017.06.091

Cummings EB (1995) Laser-Induced Thermal Acoustics. PhD-Thesis, California Institute of Technology, Pasadena

Cummings EB, Leyva IA, Hornung HG (1995) Laser-induced thermal acoustics (LITA) signals from finite beams. Appl Opt 34(18):3290-3302. https://doi.org/10.1364/AO.34.003290

Demizu M, Terazima M, Kimura Y (2008) Transport properties of binary mixtures of carbon dioxide and 1-butyl-3-methylimidazolium hexafluorophosphate studied by transient grating spectroscopy. Anal Sci 24:1329-1334

Eichler HJ, Günter P, Pohl DW (1986) Laser Induced Dynamic Gratings. Springer series in optical sciences ; 50, Springer, Berlin and Heidelberg and New York and Tokyo

Falgout Z, Rahm M, Sedarsky D, Linne M (2016) Gas/fuel jet interfaces under high pressures and temperatures. Fuel 168:14-21. https://doi.org/10.1016/j.fuel.2015.11.061

Förster FJ (2016) Laser-induced thermal acoustics: simultaneous velocimetry and thermometry for the study of compressible flows. PhD-Thesis, University of Stuttgart, Stuttgart

Förster FJ, Baab S, Lamanna G, Weigand B (2015) Temperature and velocity determination of shock-heated flows with non-resonant heterodyne laser-induced thermal acoustics. Applied Physics B 121(3):235-248. https://doi.org/10.1007/s00340-015-6217-7

Förster FJ, Baab S, Steinhausen C, Lamanna G, Ewart P, Weigand B (2018) Mixing characterization of highly underexpanded fluid jets with real gas expansion. Exp Fluids 59(3):6247. https://doi. org/10.1007/s00348-018-2488-1

Hemmerling B, Kozlov DN (1999) Generation and temporally resolved detection of laser-induced gratings by a single, pulsed Nd: YAG laser. Appl Opt 38(6):1001. https://doi.org/10.1364/AO.38.00100 1

Hubschmid W, Hemmerling B, Stampanoni-Panariello A (1995) Rayleigh and Brillouin modes in electrostrictive gratings. J Opt Soc Am B 12(10):1850. https://doi.org/10.1364/JOSAB.12.001850

Joint Committee for Guides in Metrology (2008) Evaluation of measurement data - Guide to the expression of uncertainty in measurement: GUM 1995 with minor corrections, 1st edn. JCGM 100:2008 
Kimura Y, Kanda D, Terazima M, Hirota N (1995) Application of the transient grating method to the measurement of transport properties for high pressure fluids. Phys Chem Chem Phys 99(2):196203. https://doi.org/10.1002/bbpc.19950990214

Kimura Y, Yamamoto Y, Fujiwara H, Terazima M (2005a) Vibrational energy relaxation of azulene studied by the transient grating method. I. Supercritical fluids. J Chem Phys 123(5):054512. https://doi.org/10.1063/1.1994847

Kimura Y, Yamamoto Y, Terazima M (2005b) Vibrational energy relaxation of azulene studied by the transient grating method. II. Liquid solvents. J Chem Phys 123(5):054513. https://doi. org/10.1063/1.1994848

Lamanna G, Steinhausen C, Weigand B, Preusche A, Bork B, Dreizler A, Stierle R, Gross J (2018) On the importance of non-equilibrium models for describing the coupling of heat and mass transfer at high pressure. Int Commun Heat Mass Transf 98:49-58. https:// doi.org/10.1016/j.icheatmasstransfer.2018.07.012

Lamanna G, Steinhausen C, Weckenmann F, Weigand B, Bork B, Preusche A, Dreizler A, Stierle R, Gross J (2020) Laboratory Experiments of High-Pressure Fluid Drops: Chapter 2. American Institute of Aeronautics and Astronautics (Hg) - High-Pressure Flows for Propulsion Applications pp 49-109, https://doi. org/10.2514/5.9781624105814.0049.0110

Latzel H, Dreier T (2000) Sound velocity, heat conduction and virial coefficients of gaseous mixtures at high pressure from NIR laserinduced grating experiments. Phys Chem Chem Phys 2(17):38193824. https://doi.org/10.1039/b003271i

Lemmon EW, Bell IH, Huber ML, McLinden MO (2018) NIST Standard Reference Database 23: Reference Fluid Thermodynamic and Transport Properties-REFPROP, Version 10.0, National Institute of Standards and Technology. https://doi.org/10.18434/T4JS3C, https://www.nist.gov/srd/refprop

Li Y, Roberts WL, Brown MS (2002) Investigation of gaseous acoustic damping rates by transient grating spectroscopy. AIAA J 40(6):1071-1077. https://doi.org/10.2514/2.1790

Li Y, Roberts WL, Brown MS, Gord JR (2005) Acoustic damping rate measurements in binary mixtures of atomic species via transientgrating spectroscopy. Exp Fluids 39(4):687-693. https://doi. org/10.1007/s00348-005-1012-6

Meier K, Laesecke A, Kabelac S (2004) Transport coefficients of the Lennard-Jones model fluid. I. Viscosity. J Chem Phys 121(8):3671-3687. https://doi.org/10.1063/1.1770695

Meier K, Laesecke A, Kabelac S (2005) Transport coefficients of the Lennard-Jones model fluid. III. Bulk viscosity. J Chem Phys 122(1):14513. https://doi.org/10.1063/1.1828040

Moffat RJ (1988) Describing the uncertainties in experimental results. Exp Thermal Fluid Sci 1(1):3-17. https://doi.org/10.1016/08941777(88)90043-X

Müller H, Niedermeier CA, Matheis J, Pfitzner M, Hickel S (2016) Large-eddy simulation of nitrogen injection at trans- and supercritical conditions. Phys Fluids 28(1):015102. https://doi. org/10.1063/1.4937948
Mysik SV (2015) Analyzing the acoustic spectra of sound velocity and absorption in amphiphilic liquids. St Petersburg Polytech Univ J 1(3):325-331. https://doi.org/10.1016/j.spjpm.2015.12.003

Nomura H, Nakaya S, Tsue M (2020) Microgravity Research on QuasiSteady and Unsteady Combustion of Fuel Droplet at High Pressures: Chapter 1. American Institute of Aeronautics and Astronautics $(\mathrm{Hg})$ - High-Pressure Flows for Propulsion Applications pp 1-47, https://doi.org/10.2514/5.9781624105814.0001.0048

Powers PE (2013) Field guide to nonlinear optics, SPIE field guide series, vol FG29. SPIE Press, Bellingham Washington USA

Qiao L, Jain S, Mo G (2020) Molecular Simulations to Research Supercritical Fuel Properties: Chapter 10. American Institute of Aeronautics and Astronautics ( $\mathrm{Hg}$ ) - High-Pressure Flows for Propulsion Applications pp 409-460, https://doi.org/10.2514/5.97816 24105814.0409.0460

Santoro M, Gorelli FA (2008) Structural changes in supercritical fluids at high pressures. Physical Review B 77(21), https://doi. org/10.1103/PhysRevB.77.212103

Schlamp S, Cummings EB, Hornung HG (1999) Beam misalignments and fluid velocities in laser-induced thermal acoustics. Appl Opt 38(27):5724. https://doi.org/10.1364/AO.38.005724

Siegman AE (1977) Bragg diffraction of a Gaussian beam by a crossedGaussian volume grating. J Opt Soc Am 67(4):545-550

Simeoni GG, Bryk T, Gorelli FA, Krisch M, Ruocco G, Santoro M, Scopigno T (2010) The Widom line as the crossover between liquid-like and gas-like behaviour in supercritical fluids. Nat Phys 6(7):503-507. https://doi.org/10.1038/nphys 1683

Stampanoni-Panariello A, Kozlov DN, Radi PP, Hemmerling B (2005a) Gas phase diagnostics by laser-induced gratings I. theory. Applied Physics B 81(1):101-111, https://doi.org/10.1007/s0034 $0-005-1852-z$

Stampanoni-Panariello A, Kozlov DN, Radi PP, Hemmerling B (2005b) Gas-phase diagnostics by laser-induced gratings II. experiments. Appl Phys B 81(1):113-129. https://doi.org/10.1007/ s00340-005-1853-y

Steinhausen C, Reutzsch J, Lamanna G, Weigand B, Stierle R, Gross J, Preusche A, Dreizler A (2019) Droplet Evaporation under High Pressure and Temperature Conditions: A Comparison of Droplet Evaporation under High Pressure and Temperature Conditions: A Comparison of Experimental Estimations and Direct Numerical Simulations. Proceedings ILASS-Europe, (2019) 29th Conference on Liquid Atomization and Spray Systems: 2-4 September 2019. France, Paris

Stierle R, Waibel C, Gross J, Steinhausen C, Weigand B, Lamanna G (2020) On the selection of boundary conditions for droplet evaporation and condensation at high pressure and temperature conditions from interfacial transport resistivities. Int J Heat Mass Transf 151:119450. https://doi.org/10.1016/j.ijheatmasstrans fer.2020.119450

Publisher's Note Springer Nature remains neutral with regard to jurisdictional claims in published maps and institutional affiliations.

\section{Affiliations}

\section{Christoph Steinhausen ${ }^{1}\left[\begin{array}{l}\text { D } \\ \text { Valerie Gerber }\end{array}{ }^{1}\right.$. Andreas Preusche ${ }^{2}$. Bernhard Weigand ${ }^{1}$. Andreas Dreizler ${ }^{2}$. Grazia Lamanna ${ }^{1}$}

\section{Christoph Steinhausen}

christoph.steinhausen@itlr.uni-stuttgart.de

1 Institute of Aerospace Thermodynamics (ITLR), University of Stuttgart, Pfaffenwaldring 31, 70569 Stuttgart, Germany
2 Institute Reactive Flows and Diagnostics (RSM), Technical University of Darmstadt, Otto-Berndt-Str. 3, 64287 Darmstadt, Germany 